\title{
Quantum order-by-disorder driven phase reconstruction in the vicinity of ferromagnetic quantum critical points
}

\author{
Una Karahasanovic, ${ }^{1}$ Frank Krüger, ${ }^{1}$ and Andrew G. Green ${ }^{2}$ \\ ${ }^{1}$ SUPA, School of Physics and Astronomy, University of St. Andrews, North Haugh, St. Andrews KY16 9SS, United Kingdom \\ ${ }^{2}$ London Centre for Nanotechnology, University College London, Gordon Street, London WC1H OAH, United Kingdom
}

(Received 18 January 2012; revised manuscript received 22 March 2012; published 6 April 2012)

\begin{abstract}
The formation of new phases close to itinerant electron quantum critical points has been observed experimentally in many compounds. We present a unified analytical framework to explain the emergence of new types of order around itinerant ferromagnetic quantum critical points. The central idea of our analysis is that certain Fermi-surface deformations associated with the onset of the competing order enhance the phase space available for low-energy quantum fluctuations and so, self-consistently lower the free energy. We demonstrate that this quantum order-by-disorder mechanism leads to instabilities toward the formation of spiral and $d$-wave spin-nematic phases close to itinerant ferromagnetic quantum critical points in three spatial dimensions.
\end{abstract}

DOI: 10.1103/PhysRevB.85.165111

PACS number(s): $74.40 . \mathrm{Kb}, 75.30 . \mathrm{Kz}, 71.10 .-\mathrm{w}$

\section{INTRODUCTION}

A variety of unusual phenomena that do not fit into the framework of Fermi-liquid theory have been observed in the vicinity of quantum phase transitions. An interesting example is the emergence of new phases near putative quantum critical points, observed in many experiments. Examples include the onset of superconductivity close to the itinerant ferromagnetic (FM) quantum critical point of $\mathrm{UGe}_{2},{ }^{1,2}$ an anomalous anisotropic phase around the metamagnetic quantum critical end point of $\mathrm{Sr}_{3} \mathrm{Ru}_{2} \mathrm{O}_{7}, 3,4$ a possible inhomogeneous magnetic state in $\mathrm{ZrZn}_{2},{ }^{5}$ or the unusual partially ordered phase of $\mathrm{MnSi}^{6}{ }^{6}$ This has led to the speculation that the onset of new phases close to quantum critical points might represent a generic principle. ${ }^{7}$

In his pioneering paper, Hertz $^{8}$ studied the paramagnetto-ferromagnet quantum phase transition of itinerant fermions that occurs by varying the exchange coupling between electron spins. He derived an effective action for dynamical fluctuations of the bosonic-order parameter. Later, Millis ${ }^{9}$ used this approach to calculate temperature dependencies of the correlation length, susceptibility, and specific heat. In the past decade, several authors ${ }^{10-15}$ carried out diagrammatic calculations that extend beyond Hertz-Millis theory. They showed that the free energy contains a nonanalytic dependence on the order parameter and its gradients. It was argued ${ }^{10}$ that nonanalytic terms occur due to additional soft particle-hole modes that couple to the order-parameter fluctuations. These nonanalytic corrections can render the transitions weakly first order at low temperatures and can lead to the instability of quantum critical points for the formation of new phases.

We present an alternative analytical approach—quantum order by disorder - which is able to predict new phases and provides an intuitive physical picture of the problem. Moreover, it results in relatively simple calculations, accessible to a broad audience. It relies on the idea that certain deformations of the Fermi surface enhance the phase space available for low-energy quantum fluctuations and, thus, self-consistently lower the free energy. This results in new phases near the putative quantum critical point. Note that, in the familiar realizations, ${ }^{16-19}$ the quantum order-by-disorder mechanism is driven by bosonic-order-parameter fluctuations. Here, the underlying fermionic statistics of the excitations and Pauli blocking of the phase space are very important. Recently, it has been demonstrated ${ }^{20}$ that the formation of the enigmatic partially ordered phase of MnSi (Ref. 6) with its peculiar magnetic-ordering pattern can be explained by the fermionic quantum order-by-disorder mechanism.

Our paper builds upon the work of Conduit et al. ${ }^{21}$ in which a spiral phase was predicted close to the itinerant ferromagnetic quantum critical point in three spatial dimensions. In that paper, the quantum order-by-disorder approach was used with a numerical evaluation of the fluctuation corrections to the free energy in the presence of a spiral state. We develop an analytical approach that ultimately allows us to extend the framework to include new phases, such as the spin-nematic and a Pomeranchuk-type instability in which the net magnetization is absent, and the Fermi-surface deformations for spin-up and spin-down electrons are of opposite signs. ${ }^{22-24}$

This is achieved through a Ginzburg-Landau expansion in the vicinity of the finite-temperature tricritical point. We calculate closed expressions for the Ginzburg-Landau coefficients of a uniform ferromagnet and evaluate them analytically in the low-temperature limit. The Ginzburg-Landau coefficients of the spiral ferromagnet are related to the coefficients of the uniform ferromagnet by averages of certain angular functions.

For the spin nematic, we first develop an expansion of the generating function in powers of the field conjugate to the spin-nematic-order parameter. Similarly, the coefficients in this expansion are related to the Ginzburg-Landau coefficients of the uniform ferromagnet by averages of certain angular functions. A Legendre transform of the generating function recovers the expression for the free energy. By including small deviations from the isotropic free-electron dispersion, we are able to obtain phase diagrams relevant to a broader range of experimental systems.

This paper is organized as follows: In Sec. II, we discuss the key ideas of quantum order by disorder and outline its mathematical setting. In Sec. III, we proceed to construct the Ginzburg-Landau expansions for the uniform ferromagnetic, spin-spiral, and spin-nematic states. This enables us to construct the phase diagram in Sec. IV. Finally, in Sec. V, we summarize our results and suggest directions for future papers. 


\section{QUANTUM ORDER BY DISORDER}

The central idea of quantum order by disorder is that certain phases have more low-energy quantum fluctuations associated with them. This lowers their free energy and renders them stable. The effect of the lowering of the free energy already becomes evident in second-order perturbation theory; the second-order contribution to the free energy of the ground state is always negative. The mechanism is similar in some ways to the entropic lowering of the free energy in classical systems. Equivalent results for the contribution of quantum fluctuations to the free energy can be derived starting from a functional integral approach.

A well-known example of quantum order by disorder is that of a quantum antiferromagnet. If the electron spins are oriented ferromagnetically, no virtual electron hopping is allowed due to the Pauli exclusion principle. On the other hand, when the spins are antiparallel, electron hopping is allowed. This hopping lowers the free energy of the system through second-order perturbation theory. In this way, the antiferromagnetic phase is stabilized due to the effect of quantum fluctuations.

\section{A. Perturbation theory}

Let us now begin to develop this general heuristic picture into an explicit calculation. Our starting point is the free-electron system in $d=3$ spatial dimensions interacting through Hubbard point repulsion,

$$
\mathcal{H}=\sum_{\mathbf{k}, \sigma= \pm}\left(\epsilon_{\mathbf{k}}-\mu\right) \hat{n}_{\mathbf{k}, \sigma}+g \int d^{3} \mathbf{r} \hat{n}_{+}(\mathbf{r}) \hat{n}_{-}(\mathbf{r}) .
$$

Here, $\epsilon_{\mathbf{k}}=\frac{k^{2}}{2}$ is the isotropic free-electron dispersion, $\mu$ denotes the chemical potential, and $\hat{n}_{ \pm}(\mathbf{r})$ are the density operators of spin-up/-down electrons. Note that later on, we will include small anisotropic deformations to make the dispersion more tight bindinglike. The strength of the contact interaction is given by $g$. The mean-field free energy is given by

$$
\mathcal{F}_{\mathrm{MF}}=-\frac{1}{\beta} \sum_{\mathbf{k}, \sigma} \ln \left(1+e^{-\beta\left(\epsilon_{\mathbf{k}-\mu}^{\sigma}\right)}\right)+g \int d^{3} \mathbf{r} \mathbf{M}^{2}(\mathbf{r}),
$$

where $\beta=T^{-1}$ represents the inverse temperature, $\epsilon_{\mathbf{k}}^{\sigma}$ is the mean-field dispersion in the presence of a certain type of order, and $\mathbf{M}(\mathbf{r})$ is the magnetization vector. In this paper, we will not consider phases with spatial charge modulations.

The effects of fluctuations are already seen in self-consistent second-order perturbation theory. The fluctuation corrections to the free energy are given by

$$
\tilde{\mathcal{F}}_{\mathrm{fl}}=-2 g^{2} \sum_{\mathbf{k}_{1} \cdots \mathbf{k}_{4}}^{\prime} \frac{n_{\mathbf{k}_{1}}^{+} n_{\mathbf{k}_{2}}^{-}\left(1-n_{\mathbf{k}_{3}}^{+}\right)\left(1-n_{\mathbf{k}_{4}}^{-}\right)}{\epsilon_{\mathbf{k}_{1}}^{+}+\epsilon_{\mathbf{k}_{2}}^{-}-\epsilon_{\mathbf{k}_{3}}^{+}-\epsilon_{\mathbf{k}_{4}}^{-}},
$$

where the prime over the sum indicates momentum conservation $\mathbf{k}_{1}+\mathbf{k}_{2}=\mathbf{k}_{3}+\mathbf{k}_{4}$ and for brevity, we have written the Fermi functions as

$$
n_{\mathbf{k}}^{\sigma}:=n\left(\epsilon_{\mathbf{k}}^{\sigma}\right)=\left(e^{\beta\left(\epsilon_{\mathbf{k}}^{\sigma}-\mu\right)}+1\right)^{-1} .
$$

(a)

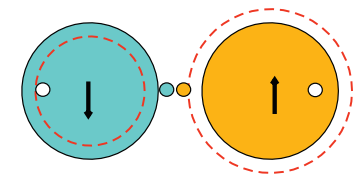

(b)

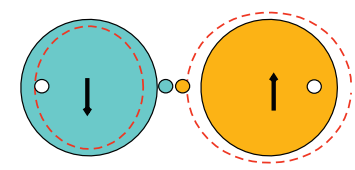

(c)

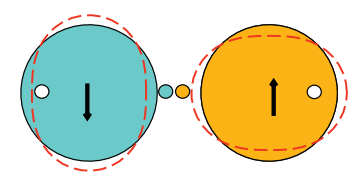

FIG. 1. (Color online) Distortions of the Fermi surface (dashed lines) enhance the phase space for quantum fluctuations. (a) Uniform ferromagnet, (b) spiral, and (c) $d$-wave spin nematic. Quantum fluctuations correspond to excitations of pairs of particle-hole pairs of opposite spin and equal and opposite momenta.

Note that the fluctuation corrections to the free energy are calculated self-consistently; the energies entering the Fermi functions are the mean-field dispersions in the presence of a given type of order.

From Eq. (3), we see that the fluctuations correspond to excitations of virtual pairs of particle-hole pairs of opposite spin and equal and opposite momenta (spin-up particle-hole pairs carry momentum $\mathbf{k}_{1}-\mathbf{k}_{3}$, and spin-down particle-hole pairs carry momentum $\mathbf{k}_{2}-\mathbf{k}_{4}$ ). Since we need to put in energy to create the particle-hole pairs, the denominator of Eq. (3) is always positive, which results in negative contributions to the free energy. Certain deformations of the Fermi surface enhance the phase space available for virtual low-energy particle-hole excitations and, in that way, self-consistently stabilize new phases. Ferromagnetic, spiral, or spin-nematic Fermi-surface distortions, which are shown schematically in Fig. 1, all open up extra phase spaces for the low-energy particle-hole pairs to form.

Careful inspection of Eq. (3) reveals that the term,

$$
\tilde{\mathcal{F}}_{\mathrm{fl}}^{\infty}=-2 g^{2} \sum_{\mathbf{k}_{1} \cdots \mathbf{k}_{4}}^{\prime} \frac{n_{\mathbf{k}_{1}}^{+} n_{\mathbf{k}_{2}}^{-}}{\epsilon_{\mathbf{k}_{1}}^{+}+\epsilon_{\mathbf{k}_{2}}^{-}-\epsilon_{\mathbf{k}_{3}}^{+}-\epsilon_{\mathbf{k}_{4}}^{-}}
$$

contained in $\tilde{\mathcal{F}}_{\text {fl }}$, gives an unphysical divergent contribution to the free energy. To avoid this, we need to take into account the renormalization of the interaction matrix element $g .{ }^{25}$ We perform a self-consistent perturbative expansion around a mean-field solution. Instead of using the momentum to label the eigenstates of the free-electron Hamiltonian, we use it to label first-order shifted states,

$$
\begin{aligned}
|\mathbf{k} \uparrow, \mathbf{I} \downarrow\rangle= & |\mathbf{k} \uparrow, \mathbf{I} \downarrow\rangle_{0} \\
& +\sum_{\mathbf{p} \neq \mathbf{k}, \mathbf{q} \neq \mathbf{l}} \frac{0\left\langle\mathbf{p} \uparrow, \mathbf{q} \downarrow\left|\mathcal{H}^{\text {int }}\right| \mathbf{k} \uparrow, \mathbf{I} \downarrow\right\rangle_{0}}{\epsilon_{\mathbf{k}}^{+}+\epsilon_{\mathbf{l}}^{-}-\epsilon_{\mathbf{p}}^{+}-\epsilon_{\mathbf{q}}^{-}}|\mathbf{p} \uparrow, \mathbf{q} \downarrow\rangle_{0},
\end{aligned}
$$

where $|\mathbf{k} \uparrow, \mathbf{I} \downarrow\rangle_{0}$ labels the two-particle free-electron state and $|\mathbf{k} \uparrow, \mathbf{I} \downarrow\rangle$ labels the two-particle state corrected to first order in 
the interaction $g . \epsilon_{\mathbf{k}}^{\sigma}$ are taken self-consistently to be the meanfield electron dispersions, and $\mathcal{H}^{\text {int }}$ represents the interaction Hamiltonian. With this identification, we must also make a corresponding alteration to the matrix element $g$,

$$
g_{\mathbf{k}_{1}, \mathbf{k}_{2}} \rightarrow g-2 g^{2} \sum_{\mathbf{k}_{3}, \mathbf{k}_{4}}^{\prime} \frac{1}{\epsilon_{\mathbf{k}_{1}}^{+}+\epsilon_{\mathbf{k}_{2}}^{-}-\epsilon_{\mathbf{k}_{3}}^{+}-\epsilon_{\mathbf{k}_{4}}^{-}} .
$$

This renormalization leads to a regular expression for the free energy,

$$
\mathcal{F}_{\mathrm{fl}}=2 g^{2} \sum_{\mathbf{k}_{1} \cdots \mathbf{k}_{4}}^{\prime} \frac{n_{\mathbf{k}_{1}}^{+} n_{\mathbf{k}_{2}}^{-}\left(n_{\mathbf{k}_{3}}^{+}+n_{\mathbf{k}_{4}}^{-}\right)}{\epsilon_{\mathbf{k}_{1}}^{+}+\epsilon_{\mathbf{k}_{2}}^{-}-\epsilon_{\mathbf{k}_{3}}^{+}-\epsilon_{\mathbf{k}_{4}}^{-}} .
$$

\section{B. Functional integral derivation}

We next sketch how the same result can be derived using field-theoretical methods. ${ }^{21,26}$ This approach reveals the connection between self-consistent second-order perturbation theory, outlined above, and field-theoretical calculations that explicitly show nonanalytic behavior of the free energy. We start from the fermionic partition function,

$$
\begin{aligned}
\mathcal{Z} & =\int \mathcal{D}(\bar{\psi}, \psi) e^{-\mathcal{S}[\bar{\psi}, \psi]}, \\
\mathcal{S}[\bar{\psi}, \psi] & =\int_{0}^{\beta} d \tau \int d^{3} \mathbf{r}\left[\bar{\psi} \partial_{\tau} \psi+\mathcal{H}(\bar{\psi}, \psi)\right],
\end{aligned}
$$

where $\psi=\left(\psi_{+}, \psi_{-}\right)^{T}$ and $\bar{\psi}=\left(\bar{\psi}_{+}, \bar{\psi}_{-}\right)$denote Grassman fields which vary throughout space and imaginary time and the Hamiltonian is given by Eq. (1). After performing a HubbardStratonovich decoupling of the interaction in spin $(\phi)$ and charge $(\rho)$ channels, we obtain

$$
\begin{aligned}
\mathcal{Z}= & \int \mathcal{D}(\bar{\psi}, \psi) \mathcal{D} \boldsymbol{\phi} \mathcal{D} \rho e^{-\mathcal{S}[\bar{\psi}, \psi, \boldsymbol{\phi}, \rho]}, \\
\mathcal{S}[\bar{\psi}, \psi, \boldsymbol{\phi}, \rho]= & \int \bar{\psi}\left[\hat{G}_{0}^{-1}+g(\rho-\boldsymbol{\phi} \cdot \boldsymbol{\sigma})\right] \psi \\
& +g \int\left(\boldsymbol{\phi}^{2}-\rho^{2}\right),
\end{aligned}
$$

where $\hat{G}_{0}^{-1}$ is the free-electron Green's function and $\sigma$ denotes the vector of Pauli matrices. Integrating out the fermions, we obtain

$$
\begin{aligned}
\mathcal{Z} & =\int \mathcal{D} \boldsymbol{\phi} \mathcal{D} \rho e^{-\mathcal{S}[\boldsymbol{\phi}, \rho]}, \\
\mathcal{S}[\boldsymbol{\phi}, \rho] & =-\operatorname{Tr} \ln \left[\hat{G}_{0}^{-1}+g(\rho-\boldsymbol{\sigma} \cdot \boldsymbol{\phi})\right]+g \int\left(\boldsymbol{\phi}^{2}-\rho^{2}\right) .
\end{aligned}
$$

So far, all the steps are the same as in Hertz-Millis theory. However, in that case, the aim was to derive an effective action for dynamical fluctuations of the bosonic-order parameter in the paramagnetic state. In contrast, we wish to derive a Ginzburg-Landau expansion in the static-order parameter. In order to do this, we separate $\phi$ and $\rho$ into zero-frequency $\left(\rho_{0}, \mathbf{M}\right)$ and finite-frequency parts $(\tilde{\rho}, \tilde{\boldsymbol{\phi}}): \rho=\rho_{0}+\tilde{\rho}$ and
$\boldsymbol{\phi}=\mathbf{M}+\tilde{\boldsymbol{\phi}}$. The action then becomes

$$
\begin{aligned}
\mathcal{S}[\phi, \rho]= & -\operatorname{Tr} \ln \left[\hat{G}_{0}^{-1}+g \rho_{0}-g \boldsymbol{\sigma} \cdot \mathbf{M}+g(\tilde{\rho}-\tilde{\boldsymbol{\phi}} \cdot \boldsymbol{\sigma})\right] \\
& +g \int\left(\mathbf{M}^{2}+\tilde{\boldsymbol{\phi}}^{2}-\tilde{\rho}^{2}\right) .
\end{aligned}
$$

We expand this action to quadratic order in finite-frequency fluctuations and integrate them out, yielding the following expression for the free energy:

$$
\begin{aligned}
\mathcal{F}[\mathbf{M}]= & g M^{2}-\operatorname{Tr} \ln \hat{G}^{-1} \\
& +\underbrace{\frac{1}{2} \operatorname{Tr} \ln \left(1+2 g \Pi^{+-}+g^{2} \Pi^{+-} \Pi^{-+}\right)}_{\mathcal{F}_{\mathrm{fl}, \perp}} \\
& +\underbrace{\frac{1}{2} \operatorname{Tr} \ln \left(1-g^{2} \Pi^{++} \Pi^{--}\right)}_{\mathcal{F}_{\mathrm{ff}, \|}} .
\end{aligned}
$$

The term $\mathcal{F}_{\mathrm{fl}, \perp}$ arises from transverse magnetic fluctuations, while the term $\mathcal{F}_{\mathrm{fl}, \|}$ accounts for charge-density and longitudinal magnetic fluctuations. The polarization bubbles are given by

$$
\Pi^{\sigma \sigma^{\prime}}(\mathbf{q}, \omega)=\frac{1}{\beta} \sum_{\mathbf{k}, \omega^{\prime}} \hat{G}_{\sigma}\left(\mathbf{k}, \omega^{\prime}\right) \hat{G}_{\sigma^{\prime}}\left(\mathbf{k}-\mathbf{q}, \omega^{\prime}-\omega\right),
$$

where $\hat{G}_{\sigma}^{-1}=\hat{G}_{0}^{-1}+g \rho_{0}-\sigma g M$. The polarization bubbles $\Pi^{\sigma \sigma^{\prime}}$ explicitly depend on the interaction strength $g$.

Next, we perform a rather strange expansion in $g$. We expand the Tr $\ln$ terms to second order in $g$ by only expanding in powers of $g$ that stand in front of the polarization bubbles, while keeping the full $g$ dependence of the polarization bubbles as it is. This looks like a second-order expansion in $g$, but self-consistency actually implies resummation of certain classes of contributions to infinite order. This expansion captures the relevant physics, as we will see later on. After performing the summations over Matsubara frequencies, we arrive at the expression Eq. (3). Furthermore, we need to renormalize $g$ to cancel the ultraviolet divergence. Doing so according to Eq. (7) recovers expression Eq. (8) for the free energy.

To summarize, quantum order by disorder provides a physical picture for the formation of new phases due to quantum fluctuations. As we see later, certain deformations of the Fermi surface enhance the phase space available for quantum fluctuations and, in that way, lower the free energy. This is already apparent in second-order perturbation theory and can be derived from a functional integral approach. Next, we want to expand the free energy in powers of the order parameter (which enters through the mean-field dispersion). This will enable us to construct the Ginzburg-Landau expansion and to obtain the phase diagram.

\section{GINZBURG-LANDAU EXPANSION}

We wish to determine the phase diagram of the near-critical itinerant ferromagnet allowing for the generation of new phases near the quantum critical point. In order to obtain the phase diagram, we develop a Ginzburg-Landau expansion of the free energy in powers of the order parameters for the various types of phases that might form. The expansion is valid 
in the vicinity of the tricritical point (explained later), where the value of the order parameters is sufficiently small.

It turns out that our task is simplified considerably by relationships between the expansion coefficients for the different types of order and those for the uniform ferromagnet. We begin, therefore, with an explicit evaluation of the coefficients for the uniform ferromagnet.

Next, we allow for spatial modulations of the ferromagnetic order; in particular, we consider a spiral modulation of the magnetization. We use the fact that the free energy can be expressed (for all orders) as a functional of the mean-field electron dispersion in the presence of the spiral order. We show how the coefficients of the expansion in the spiral-ordering wave vector $\mathbf{Q}$ are related (by angular averages of certain functions) to those of the uniform ferromagnet.

For other order parameters that cannot be introduced by a mean-field decoupling of the electron-electron interaction, we introduce a field conjugate to the order parameter and construct an expansion of the generating function in terms of the conjugate field. We are able to relate the coefficients of the generating function to the Ginzburg-Landau coefficients of the uniform ferromagnet by performing some simple angular integrals. We use a Legendre transformation of the generating function to recover the Ginzburg-Landau expansion of the free energy. Quantum fluctuations generate an interaction in the new channel. A similar mechanism is well known in spin-fluctuation theory where superconductivity is mediated through spin fluctuations. ${ }^{27,28}$ In what follows, we will concentrate on the case of a $d$-wave spin nematic.

Finally, we allow for a more generic energy dispersion by considering small anisotropic deviations from the isotropic free-electron dispersion. We calculate the corrections to the coefficients of the Ginzburg-Landau expansion due to the anisotropic distortion. The coefficients of this expansion are proportional to parts of the Ginzburg-Landau coefficients of the uniform ferromagnet in the presence of an isotropic dispersion. The proportionality factors are determined by angular averages of functions that encode the specific form of the deviation from the isotropic free-electron dispersion.

\section{A. Uniform ferromagnet}

The dispersion of the uniform ferromagnet is given by $\epsilon^{\sigma}(\mathbf{k})=\frac{\mathbf{k}^{2}}{2}-\sigma g M$. We Taylor expand the free energy in powers of $M$,

$$
\mathcal{F}[M]=\alpha M^{2}+\beta M^{4}+\gamma M^{6}+\cdots,
$$

where the Ginzburg-Landau coefficients $\alpha, \beta, \gamma$ are functions of the interaction strength $g$ and temperature.

\section{Mean-field coefficients}

The expansion of the mean-field free energy, Eq. (2), in powers of $M$ leads to the following coefficients:

$$
\begin{aligned}
& \alpha_{\mathrm{MF}}=g+g^{2} \sum_{\mathbf{k}} n^{(1)}\left(\epsilon_{\mathbf{k}}\right), \\
& \beta_{\mathrm{MF}}=\frac{2}{4 !} g^{4} \sum_{\mathbf{k}} n^{(3)}\left(\epsilon_{\mathbf{k}}\right),
\end{aligned}
$$

$$
\gamma_{\mathrm{MF}}=\frac{2}{6 !} g^{6} \sum_{\mathbf{k}} n^{(5)}\left(\epsilon_{\mathbf{k}}\right),
$$

where $n^{(j)}(\epsilon)=\partial_{\epsilon}^{j} n(\epsilon)$ denotes the $j$ th derivative of the Fermi function with respect to its energy argument. The remaining integrals over derivatives of Fermi functions are straightforward to calculate for the present $k^{2}$ dispersion.

\section{Fluctuation contributions to coefficients}

Here, we outline the main steps in the calculation of fluctuation contributions to the Ginzburg-Landau coefficients. The detailed calculation is given in Appendix B. We start by writing the fluctuation corrections to the free energy in terms of modified particle-hole densities of states Eq. (A1). This is possible because the fluctuations correspond to excitations of virtual pairs of particle-hole pairs. The particle-hole densities of states can be calculated analytically as functions of temperature and magnetization [see Appendix A]. We can write down the expression for the fluctuation contributions to the Ginzburg-Landau coefficients $\alpha_{\mathrm{fl}}$ and $\beta_{\mathrm{fl}}$ in terms of integrals over the modified particle-hole densities of states and their derivatives with respect to the magnetization [see Appendix B]. The fluctuation corrections $\alpha_{\mathrm{fl}}$ and $\beta_{\mathrm{fl}}$ are calculated analytically at low temperatures and are calculated numerically over the full temperature range. In this paper, the phase diagrams are calculated using only the mean-field contribution to the sixth-order coefficient $\gamma$ since it is a higher-order term in the Ginzburg-Landau expansion and since the fluctuation corrections to $\gamma$ are extremely difficult to compute. At low temperatures, the fluctuation contributions to the coefficients are given by

$$
\begin{aligned}
& \alpha_{\mathrm{fl}} \simeq-\lambda(1+2 \ln 2) g^{4}, \\
& \beta_{\mathrm{fl}} \simeq \lambda\left(1+\ln \frac{T}{\mu}\right) g^{6},
\end{aligned}
$$

with $\lambda=[16 \sqrt{2}] /\left[3(2 \pi)^{6}\right]$. Note that, here and in the following, $g$ is given in dimensionless units. The $\ln (T / \mu)$ dependence of $\beta$ is a remnant of the $M^{4} \ln \left[M^{2}+(T / \mu)^{2}\right]$ term in the free energy of Vojta et al. ${ }^{11}$

\section{B. Spiral}

Next, we calculate the coefficients of the Ginzburg-Landau expansion allowing for a spatial modulation of the magnetic order. We restrict our consideration to a single planar spiral. We exploit the fact that the free energy is a functional of the mean-field dispersion in the presence of the spiral magnetic order.

\section{Mean-field dispersion in the presence of spiral magnetic order}

First, we determine the mean-field dispersion in the presence of spiral magnetic order. Let the directrix of the spiral wave vector point along the $z$ direction. For a planar spiral, the magnetization vector is then restricted to lie in the $x y$ plane, $\mathbf{M}(\mathbf{r})=M[\cos (2 \mathbf{Q} \cdot \mathbf{r}), \sin (2 \mathbf{Q} \cdot \mathbf{r}), 0]$. Note that the Hamiltonian given by Eq. (1) does not favor a particular direction of the spiral. The mean-field Hamiltonian is then 
given by

$$
\mathcal{H}=\sum_{\mathbf{k}} \tilde{\psi}_{\mathbf{k}}^{\dagger}\left(\begin{array}{cc}
\epsilon_{\mathbf{k}+\mathbf{Q}} & g M \\
g M & \epsilon_{\mathbf{k}-\mathbf{Q}}
\end{array}\right) \tilde{\psi}_{\mathbf{k}}+g M^{2},
$$

where

$$
\tilde{\psi}_{\mathbf{k}}^{\dagger}=\left(\psi_{\mathbf{k}+\mathbf{Q},+}^{\dagger}, \psi_{\mathbf{k}-\mathbf{Q},-}^{\dagger}\right)
$$

Diagonalization of this Hamiltonian leads to the mean-field dispersion,

$$
\begin{aligned}
\epsilon_{\mathbf{k}}^{\sigma}= & \frac{\epsilon_{\mathbf{k}-\mathbf{Q}}+\epsilon_{\mathbf{k}+\mathbf{Q}}}{2} \\
& -\sigma \sqrt{\left(\frac{\epsilon_{\mathbf{k}-\mathbf{Q}}-\epsilon_{\mathbf{k}+\mathbf{Q}}}{2}\right)^{2}+(g M)^{2}} .
\end{aligned}
$$

For the case of a quadratic dispersion, this reduces to

$$
\epsilon_{\mathbf{k}}^{\sigma}=\frac{k^{2}}{2}-\sigma \sqrt{(\mathbf{k} \cdot \mathbf{Q})^{2}+(g M)^{2}} .
$$

We see that the spiral wave vector enters the energy dispersion as an angle-dependent magnetization. Note that we have absorbed a $Q^{2}$ term into the chemical potential.

\section{Mean-field Ginzburg-Landau coefficients}

We will now make use of this mean-field electron dispersion in the presence of a spiral in order to determine the GinzburgLandau expansion coefficients. We Taylor expand the free energy of the spiral in powers on magnetization $M$ and wave vector $Q$, keeping the terms up to sixth order,

$$
\begin{aligned}
\mathcal{F}[M, Q]= & \left(\alpha+\beta_{1} Q^{2}+\gamma_{1} Q^{4}\right) M^{2} \\
& +\left(\beta+\gamma_{2} Q^{2}\right) M^{4}+\gamma M^{6},
\end{aligned}
$$

where the coefficients $\beta_{1}, \gamma_{1}, \gamma_{2}$ remain to be determined. A useful simplification at this stage is to rescale the spiral wave vector according to $Q \rightarrow \frac{k_{F}}{g} Q$ so that it has the same dimensions as $M$. In this way, $\beta$ and $\beta_{1}$ and $\gamma, \gamma_{1}$, and $\gamma_{2}$ have the same dimensions. Let us first consider $\beta_{1}$. The mean-field contribution is given by

$$
\beta_{1, \mathrm{MF}}=2 \frac{2}{4 !} g^{4} \int_{\mathbf{k}}\left(\frac{\mathbf{k} \cdot \mathbf{Q}}{k_{F} Q}\right)^{2} n^{(3)}\left(\epsilon_{k}\right) .
$$

Since $T \ll \mu$, derivatives of Fermi functions are strongly peaked around the Fermi energy, and we can set $|\mathbf{k}|=k_{F}$ in the scalar product which leads to a simple angular weight,

$$
\frac{\mathbf{k} \cdot \mathbf{Q}}{k_{F} Q} \approx \cos \theta
$$

where $\theta$ is the angle between vectors $\mathbf{k}$ and $\mathbf{Q}$. After carrying out the angular integral, we obtain $\beta_{1, \mathrm{MF}} \approx \frac{2}{3} \beta_{\mathrm{MF}}$. Similarly, we obtain the proportionalities $\gamma_{1, \mathrm{MF}} \approx \frac{3}{5} \gamma_{\mathrm{MF}}$ and $\gamma_{2, \mathrm{MF}} \approx$ $\gamma_{\mathrm{MF}}$.

\section{Fluctuation-corrected Ginzburg-Landau coefficients}

Now, we proceed to analyze the fluctuation corrections to the expansion coefficients in Eq. (22). As in the evaluation of the mean-field coefficients, it turns out that the fluctuation contributions to the expansion coefficients in $Q$ are related to those of the uniform ferromagnet by angular factors, which are identical to those found in the mean-field case.
The fluctuation corrections to the free energy are given by an integral over momenta $\mathbf{k}_{1}, \ldots, \mathbf{k}_{4}$ of a kernel that explicitly depends on each of the momenta through the mean-field dispersion (21). The fluctuation contributions to the GinzburgLandau coefficients are obtained by differentiating Eq. (8) with respect to $M$ and $Q$. First, we differentiate the kernel with respect to the dispersion and then the dispersion with respect to $M$ and $Q$. For example, the fluctuation contribution to the $M^{2} Q^{2}$ coefficient is given by

$$
\beta_{1, \mathrm{fl}}=\left.\frac{\partial^{2} \mathcal{F}_{\mathrm{fl}}}{\partial M^{2} \partial Q^{2}}\right|_{Q=0, M=0} .
$$

We use two important facts in order to calculate this: (i) that the free energy is a functional of dispersion and (ii) that the spiral wave vector enters the mean-field dispersion Eq. (21) like an angle-dependent magnetization.

The dispersion enters for each of the momenta $\mathbf{k}_{i}$, where $i=1, \ldots, 4$, in the momentum sum in Eq. (8). Differentiating with respect to $Q^{2}$, therefore, will bring down factors of $\left[\mathbf{k}_{i} \cdot \mathbf{Q} /\left(k_{F} Q\right)\right]^{2}$, each of which will contribute with an angular factor as in the mean-field case. This leads to the proportionality $\beta_{1, \mathrm{fl}} \approx \frac{2}{3} \beta_{\mathrm{fl}}$. Combining this with the identical result for the mean-field contribution, we obtain $\beta_{1} \approx \frac{2}{3} \beta$. When the proportionality between all of the coefficients is taken into account, the free energy (22) becomes

$$
\begin{aligned}
\mathcal{F}[M, Q]= & \left(\alpha+\frac{2}{3} \beta Q^{2}+\frac{3}{5} \gamma Q^{4}\right) M^{2} \\
& +\left(\beta+\gamma Q^{2}\right) M^{4}+\gamma M^{6} .
\end{aligned}
$$

\section{Other instabilities}

Other phases, with order parameters that cannot be introduced by a Hubbard-Stratonovich mean-field decoupling of the point Hubbard interaction, are slightly more difficult to analyze. Examples include $p$-wave superconductors and spin-nematic phases. To circumvent this problem, we begin by introducing a field $h_{N}$ conjugate to the order parameter $N$. We calculate the generating function $\varphi\left[h_{N}\right]$ following the procedure outlined for the uniform ferromagnet in Sec. III A. The free energy $\mathcal{F}[N]$ is then obtained by a Legendre transform of the generating function. In the following, we will outline this for a spin nematic.

To be more specific, we introduce a $d$-wave spin-nematicorder parameter,

$$
\begin{aligned}
N & =\sum_{\mathbf{k}, \sigma} \sigma d_{\mathbf{k}} n_{\mathbf{k}, \sigma}, \\
d_{\mathbf{k}} & =\frac{1}{k_{F}^{2}}\left(k_{x}^{2}-k_{y}^{2}\right) \approx \sin ^{2}(\theta) \cos (2 \phi),
\end{aligned}
$$

where $d_{\mathbf{k}}$ is the $d$-wave distortion. The spin-nematic-order parameter looks like a magnetization weighted by an angular factor. It corresponds to Fermi-surface distortions which have opposite signs for spin-up and spin-down electrons. The net magnetization, however, vanishes since the volumes of the distorted spin-up and spin-down Fermi surfaces are the same [see Fig. 1(c)]. As we see later, it is straightforward to generalize our final results for spin-nematic states with different symmetries. 
The generating function for this spin-nematic order is obtained by introducing a fictitious field conjugate to the spin-nematic-order parameter $N$ by adding a term,

$$
\mathcal{H}_{h_{N}}=\sum_{\mathbf{k}, \sigma} h_{N} \sigma d_{\mathbf{k}} \hat{n}_{\mathbf{k}, \sigma},
$$

to the Hamiltonian Eq. (1). The electron dispersion in the presence of this field is modified to

$$
\epsilon_{\mathbf{k}}^{\sigma}=\epsilon_{\mathbf{k}}-\sigma h_{N} \sin ^{2}(\theta) \cos (2 \phi) .
$$

The partition function in the presence of field $h_{N}$ is evaluated in precisely the manner outlined in Sec. III for the uniform ferromagnet. The logarithm of this partition function is the generating function $\varphi\left[h_{N}\right]$. Explicitly, using self-consistent second-order perturbation theory, the generating function is given by

$$
\begin{aligned}
\varphi\left[h_{N}\right]= & -\frac{1}{\beta} \sum_{\mathbf{k}, \sigma} \ln \left(1+e^{-\beta\left(\epsilon_{\mathbf{k}}^{\sigma}-\mu\right)}\right) \\
& +2 g^{2} \sum_{\mathbf{k}_{1} \cdots \mathbf{k}_{4}}^{\prime} \frac{n_{\mathbf{k}_{1}}^{+} n_{\mathbf{k}_{2}}^{-}\left(n_{\mathbf{k}_{3}}^{+}+n_{\mathbf{k}_{4}}^{-}\right)}{\epsilon_{\mathbf{k}_{1}}^{+}+\epsilon_{\mathbf{k}_{2}}^{-}-\epsilon_{\mathbf{k}_{3}}^{+}-\epsilon_{\mathbf{k}_{4}}^{-}},
\end{aligned}
$$

as a functional of the dispersion Eq. (29).

Expanding the generating function $\varphi\left[h_{N}\right]$, Eq. (30), in powers of $h_{N}$,

$$
\varphi\left[h_{N}\right]=\alpha^{\varphi} h_{N}^{2}+\beta^{\varphi} h_{N}^{4}+\gamma^{\varphi} h_{N}^{6},
$$

where the superscript $\varphi$ is used to distinguish coefficients of the generating function from those of the Ginzburg-Landau expansion. Here and in the following, $h_{N}$ is rescaled by $g$. The coefficients of this expansion may be related by angular averages to those of the Landau expansion for the uniform ferromagnet. Note that, in comparing the expansion of $\varphi\left[h_{N}\right]$ with the expansion of the free energy for the ferromagnet, there is no term directly related to $g M^{2}$ since the Hubbard point interaction is local in position space and, therefore, has no weight in the spin-nematic channel.

\section{Mean-field contributions to coefficients of the generating function}

The mean-field coefficients of the generating function are given by

$$
\begin{aligned}
& \alpha_{\mathrm{MF}}^{\varphi}=g^{2}\left\langle d_{\mathbf{k}}^{2}\right\rangle \sum_{\mathbf{k}} n^{(1)}\left(\epsilon_{\mathbf{k}}\right)=\left\langle d_{\mathbf{k}}^{2}\right\rangle\left(\alpha_{\mathrm{MF}}-g\right), \\
& \beta_{\mathrm{MF}}^{\varphi}=\frac{2}{4 !} g^{4}\left\langle d_{\mathbf{k}}^{4}\right\rangle \sum_{\mathbf{k}} n^{(3)}\left(\epsilon_{\mathbf{k}}\right)=\left\langle d_{\mathbf{k}}^{4}\right\rangle \beta_{\mathrm{MF}}, \\
& \gamma_{\mathrm{MF}}^{\varphi}=\frac{2}{6 !} g^{6}\left\langle d_{\mathbf{k}}^{6}\right\rangle \sum_{\mathbf{k}} n^{(5)}\left(\epsilon_{\mathbf{k}}\right)=\left\langle d_{\mathbf{k}}^{6}\right\rangle \gamma,
\end{aligned}
$$

where $\langle\cdots\rangle=\frac{1}{4 \pi} \int_{0}^{\pi} d \theta \sin \theta \int_{0}^{2 \pi} d \phi \cdots$ denotes the angular average. The coefficients are proportional to the corresponding Ginzburg-Landau coefficients of the uniform ferromagnet; the constants of proportionality are angular averages of powers of the nematic distortion. The resulting integrals are easy to calculate, yielding $\left\langle d_{\mathbf{k}}^{2}\right\rangle=\frac{4}{15},\left\langle d_{\mathbf{k}}^{4}\right\rangle=\frac{16}{105}$, and $\left\langle d_{\mathbf{k}}^{6}\right\rangle=\frac{320}{3003}$.

\section{Fluctuation corrections to coefficients of the generating function}

As we found in the case of the spiral, the fluctuation corrections to the coefficients in the spin-nematic expansion are related to those of the uniform ferromagnet by the same angular averages as the mean-field coefficients. For example, let us consider the fluctuation contribution to the $h_{N}^{4}$ coefficient. When differentiating the fluctuation contributions in Eq. (30) with respect to $h_{N}$ four times, this brings down terms, such as $\left\langle d_{\mathbf{k}_{1}} d_{\mathbf{k}_{2}} d_{\mathbf{k}_{3}} d_{\mathbf{k}_{4}}\right\rangle$, where $\left|\mathbf{k}_{i}\right| \approx k_{F}$ since derivatives of Fermi functions are peaked around the Fermi energy. Angular averages of this type are potentially more complicated as the directions of different k's are not independent. However, the fact that the dominant contribution comes from the particlehole pairs with momenta $\left|\mathbf{k}_{1}-\mathbf{k}_{3}\right|=\left|\mathbf{k}_{2}-\mathbf{k}_{4}\right| \approx 2 k_{F}$ leads to a tremendous simplification. Within this approximation, $\mathbf{k}_{1}, \mathbf{k}_{2}, \mathbf{k}_{3}$, and $\mathbf{k}_{4}$ are either parallel or antiparallel to one another, rendering $\left\langle d_{\mathbf{k}_{1}} d_{\mathbf{k}_{2}} d_{\mathbf{k}_{3}} d_{\mathbf{k}_{4}}\right\rangle \approx\left\langle d_{\mathbf{k}}^{4}\right\rangle$. Similar arguments hold for other types of terms that appear in the expansion. Thus, for leading order, the same proportionality holds as for the mean-field coefficients, and consequently, the generating function is given by

$$
\varphi\left[h_{N}\right]=\left\langle d_{\mathbf{k}}^{2}\right\rangle(\alpha-g) h_{N}^{2}+\left\langle d_{\mathbf{k}}^{4}\right\rangle \beta h_{N}^{4}+\left\langle d_{\mathbf{k}}^{6}\right\rangle \gamma h_{N}^{6} .
$$

\section{Ginzburg-Landau expansion of the spin nematic}

In order to obtain the Ginzburg-Landau expansion of the free energy $\mathcal{F}[N]$ of the $d$-wave spin nematic, we perform the Legendre transform of the generating function,

$$
\mathcal{F}[N]=\varphi\left[h_{N}[N]\right]+h_{N} N, \quad \frac{\partial \varphi}{\partial h_{N}}=-N .
$$

Carrying out this transformation to leading order, we obtain the free-energy expansion in powers of the nematic-order parameter $N$,

$$
\mathcal{F}[N]=-\left\langle d_{\mathbf{k}}^{2}\right\rangle(\alpha-g) N^{2}+\left\langle d_{\mathbf{k}}^{4}\right\rangle \beta N^{4}+\left\langle d_{\mathbf{k}}^{6}\right\rangle \gamma N^{6} .
$$

We point out that the derivation of the free-energy functional for the spin nematic is general and is not constrained to a particular symmetry of the distortion. To obtain the free energy of a $p$-wave spin nematic, ${ }^{22-24}$ we simply replace the $d$-wave distortion $d_{\mathbf{k}}$ by the $p$-wave angular weight $p_{\mathbf{k}} \approx \cos \theta$. This leads to slightly different angular averages $\left\langle p_{\mathbf{k}}^{2}\right\rangle=\frac{1}{3},\left\langle p_{\mathbf{k}}^{4}\right\rangle=$ $\frac{1}{5}$, and $\left\langle p_{\mathbf{k}}^{6}\right\rangle=\frac{1}{7}$ and, hence, to slightly different coefficients in the Ginzburg-Landau expansion.

\section{Deviation from free-electron dispersion}

Our approach also enables us to analyze the modifications to the phase diagram in the presence of a dispersion that slightly deviates from the free-electron dispersion, $\epsilon_{\mathbf{k}}=\frac{k^{2}}{2}+\delta \epsilon_{\mathbf{k}}$. We plug this expression into the general mean-field dispersion in the presence of a spiral, Eq. (20), to obtain

$$
\epsilon_{\mathbf{k}}^{\sigma} \approx \frac{k^{2}}{2}+\delta \epsilon_{\mathbf{k}}-\sigma \sqrt{\left[\mathbf{Q} \cdot\left(\mathbf{k}+\nabla \delta \epsilon_{\mathbf{k}}\right)\right]^{2}+(g M)^{2}} .
$$

In order to calculate the corrections to the Ginzburg-Landau coefficients, we first differentiate the free energy with respect to the dispersion and then the dispersion with respect to $M$ 
and $Q$. Finally, we expand the resulting Ginzburg-Landau coefficients in powers of $\delta \epsilon_{\mathbf{k}}$, assumed small. The free energy is now given by the sum of $\mathcal{F}_{M, Q}$, Eq. (26), and the additional contribution,

$$
\begin{aligned}
\delta \mathcal{F}[M, \mathbf{Q}]= & \left(6 \beta_{\mathrm{MF}}+\frac{g^{2}}{2} \frac{\partial^{2} \alpha_{\mathrm{fl}}}{\partial^{2} \mu^{2}}\right) \frac{\left\langle\delta \epsilon_{\mathbf{k}}^{2}\right\rangle}{g^{2}} M^{2} \\
& +15 \gamma \frac{\left\langle\delta \epsilon_{\mathbf{k}}^{2}\right\rangle}{g^{2}} M^{4}+30 \gamma \frac{\left\langle\left(\frac{\mathbf{k} \cdot \mathbf{Q}}{k_{F}}\right)^{2} \delta \epsilon_{\mathbf{k}}^{2}\right\rangle}{g^{2}} M^{2} \\
& +2 \beta_{\mathrm{MF}}\left\langle\left(\frac{\mathbf{Q} \cdot \nabla \delta \epsilon_{\mathbf{k}}}{k_{F}}\right)^{2}\right\rangle M^{2},
\end{aligned}
$$

where $\langle\cdots\rangle$ denotes an angular average and we have assumed that the deviation $\delta \epsilon_{\mathbf{k}}$ from the free-electron dispersion is such that the odd-power angular averages give zero. Mixing between coefficients at different total orders in $M$ and $Q$ now occurs since the angular distortion enters in both spinsymmetric and spin-anti-symmetric ways, as opposed to the spin-anti-symmetric Fermi-surface distortion of spiral or spinnematic states in the isotropic case.

Similarly, the free energy of the spin-nematic state is now given by the sum of $\mathcal{F}[N]$, Eq. (35), and the additional term,

$$
\begin{aligned}
\delta \mathcal{F}[N]=- & \left(6 \beta_{\mathrm{MF}}+\frac{g^{2}}{2} \frac{\partial^{2} \alpha_{\mathrm{fl}}}{\partial^{2} \mu^{2}}\right) \frac{\left\langle\delta \epsilon_{\mathbf{k}}^{2} d_{\mathbf{k}}^{2}\right\rangle}{g^{2}} N^{2} \\
& +15 \gamma \frac{\left\langle\delta \epsilon_{\mathbf{k}}^{2} d_{\mathbf{k}}^{4}\right\rangle}{g^{2}} N^{4} .
\end{aligned}
$$

\section{PHASE DIAGRAM}

In the previous section, we have shown that the free energy is a functional of the mean-field dispersion. We have used this fact to develop the Ginzburg-Landau expansions for the uniform ferromagnet and the spiral ferromagnet. The derivation of the free energy of the spin nematic is more complicated since the point interaction has no weight in the spin-nematic channel and a mean-field decoupling is not possible in that case. Instead, we added a field conjugate to the nematic-order parameter, calculated the generating function, and then performed a Legendre transform to obtain the Ginzburg-Landau expansion in terms of the spin-nematicorder parameter. Finally, we considered small deviations from the isotropic free-electron dispersion and derived the resulting corrections to the free energy.

We have shown how a very useful simplification occurs in the low-temperature regime; all spiral and spin-nematic Ginzburg-Landau coefficients can be related to those of the uniform ferromagnet, which we have calculated analytically at low temperatures.

We now use the Ginzburg-Landau expansions developed in the previous section to construct the phase diagram as a function of temperature $T$ and renormalized interaction strength $g$. We minimize the Ginzburg-Landau free energy with respect to the order parameter(s) and compare the free energies of different phases. We show how quantum fluctuations stabilize the spiral and spin-nematic phases, neither of which are favored in mean-field theory. The effect of

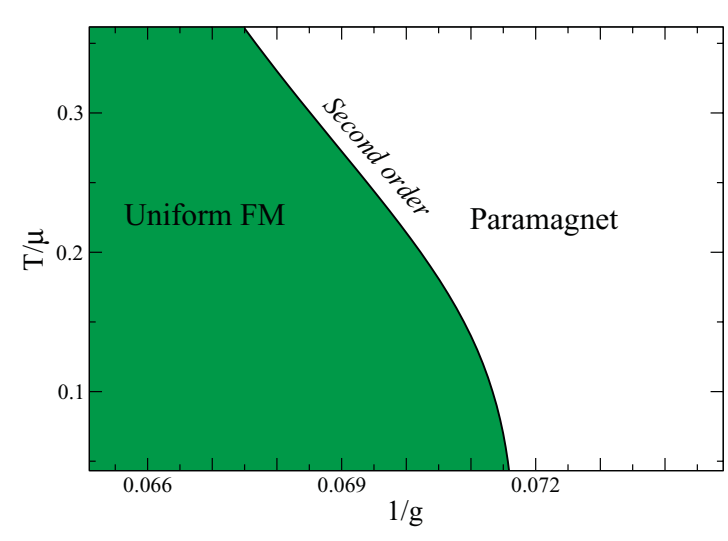

FIG. 2. (Color online) Phase diagram of the uniform ferromagnet in mean-field theory. The transition between the uniform ferromagnet and the paramagnet is always second order.

a small anisotropic correction to the free-electron dispersion on the topology of the phase diagram is also investigated.

\section{A. Mean-field phase diagram}

First, we consider the mean-field phase diagram. From Eq. (16), we see that, in the low-temperature regime, the quartic coefficient $\beta_{\mathrm{MF}}$ is positive. The second-order phase transition between paramagnetic and uniform ferromagnetic states happens when the quadratic coefficient $\alpha_{\mathrm{MF}}$ changes sign. The mean-field phase diagram is shown in Fig. 2. Neither spiral nor spin-nematic states are favored in mean-field theory.

\section{B. Phase diagram including quantum fluctuations}

Next, we include quantum fluctuations in our analysis and allow for the generation of new phases that were not present in the mean-field theory.

\section{Uniform ferromagnet}

Before investigating how fluctuations may favor the formation of new phases, we first investigate their effect on the uniform ferromagnet. From Eq. (17), we see that the fluctuations provide a negative contribution to the GinzburgLandau coefficients. Ferromagnetism is, thus, favored for lower values of the interaction strength $g$ than in the mean-field theory. This becomes evident if we consider the line of second-order transitions $\alpha=0$.

In the presence of fluctuations, the quartic coefficient $\beta$ inevitably becomes negative for low enough temperatures due to the $\sim \ln (T / \mu)$ divergence. For $\beta<0$, the paramagnetto-ferromagnet transition becomes first order. The line of first-order transitions is given by $\beta^{2}=4 \alpha \gamma$ (the condition for degeneracy of the minima of the Ginzburg-Landau function). The location of the tricritical point, at the intercept of the first-order and second-order lines, is found at $T^{*}=0.24 \mu$ (see Fig. 3). This is in good agreement with previous numerical calculations. ${ }^{21}$ The occurrence of first-order transitions at low temperatures has been observed in numerous experiments. ${ }^{5,29-33}$ 


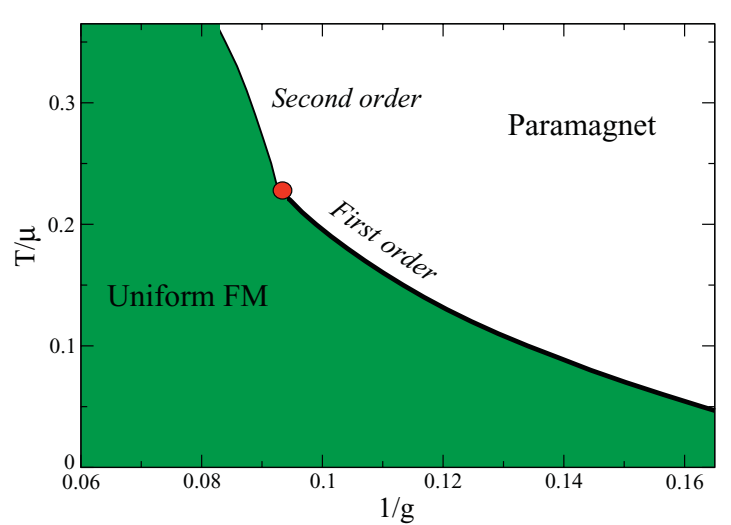

FIG. 3. (Color online) Phase diagram of the uniform ferromagnet, including quantum fluctuations. Below the tricritical point (shown as a circle), quantum fluctuations drive the transition first order.

\section{Fluctuation-driven spiral}

From Eq. (22), we see that the $Q^{2} M^{2}$ term in the free energy favors nonzero $Q$ for $\beta_{1}<0$. The particular relationship between coefficients that is found for the isotropic freeelectron dispersion implies that this occurs when $\beta<0$, i.e., the spiral first forms at the tricritical point where the transition into a uniform magnet would have become first order. The phase diagram, showing the instability toward the formation of a magnetic spiral, is shown in Fig. 4. We now derive it from the Ginzburg-Landau functional.

In the case of the isotropic free-electron dispersion, we have shown that $\beta_{1}=2 \beta / 3$. This results in the formation of a spiral state below the tricritical point. Minimizing the free-energy Eq. (26) with respect to $Q$, we obtain the optimal wave vector,

$$
\bar{Q}^{2}=-\frac{5}{6 \gamma}\left(\frac{2}{3} \beta+M^{2} \gamma\right)
$$

After substituting this value of $Q$ back in Eq. (26), we obtain the free energy at the optimal wave vector' as a function

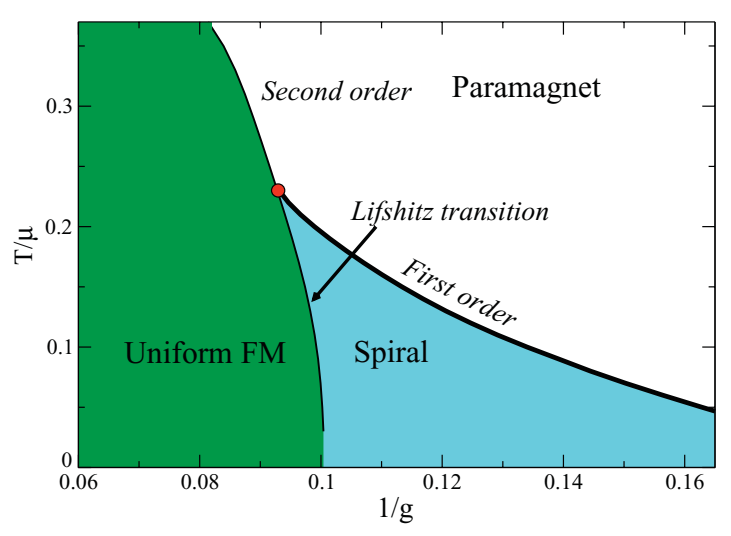

FIG. 4. (Color online) Phase diagram of the itinerant ferromagnet allowing for the possibility of spatially modulated ferromagnetism. Quantum fluctuations drive the formation of a spiral phase, which sets in below the tricritical point and preempts the first-order transition between the uniform ferromagnet and the paramagnet. of $M$,

$$
\begin{aligned}
\mathcal{F}_{\bar{Q}}[M] & =\alpha_{\bar{Q}} M^{2}+\beta_{\bar{Q}} M^{4}+\gamma_{\bar{Q}} M^{6}, \\
\alpha_{\bar{Q}} & =\left(\alpha-\frac{5}{27} \frac{\beta^{2}}{\gamma}\right), \quad \beta_{\bar{Q}}=\frac{4}{9} \beta, \quad \gamma_{\bar{Q}}=\frac{7}{12} \gamma .
\end{aligned}
$$

a. Spiral-to-paramagnet transition. In principle, there are two ways in which the system can make a transition from a paramagnet into a spiral state:

(i) A second-order transition in $M$, along which $M=0$. This line is given by $\alpha_{\bar{Q}}=0$, and the necessary condition for its existence is that $\beta_{\bar{Q}}>0$.

(ii) A first-order transition in $M$, along which $M$ changes discontinuously from zero to a finite value. This transition happens along the line $\beta_{\bar{Q}}^{2}=4 \alpha_{\bar{Q}} \gamma_{\bar{Q}}$, as long as $\beta_{\bar{Q}}<0$ and $\alpha_{\bar{Q}}>0$.

Since we have already established that we can have a spiral state only for $\beta_{1}<0$ and, hence, $\beta, \beta_{\bar{Q}}<0$ (following from the proportionalities of coefficients), we rule out the first possibility and conclude that the transition from the paramagnet into the spiral ferromagnet must be first order in $M$ [and first order in $Q$, according to Eq. (39)]. Substituting $\alpha_{\bar{Q}}, \beta_{\bar{Q}}$, and $\gamma_{\bar{Q}}$ from Eq. (40), the equation for this line becomes $\alpha \gamma=\frac{17}{63} \beta^{2}$. This transition preempts the transition from the paramagnet into the uniform ferromagnetic state [see Fig. 4].

b. Uniform ferromagnet-to-spiral transition. Next, we wish to determine the boundary between the spiral phase and the uniform ferromagnet. In principle, this may occur either discontinuously or smoothly. In the case of the free-electron dispersion, it occurs via a Lifshitz transition where $\bar{Q}$ goes continuously to zero. The value of magnetization that minimizes the free energy $\mathcal{F}_{\bar{Q}}$, Eq. (40), is given by

$$
M^{2}=\frac{-2 \beta}{7 \gamma}\left[\frac{8}{9}+\sqrt{\left(\frac{8}{9}\right)^{2}-7\left(\frac{\alpha \gamma}{\beta^{2}}-\frac{5}{27}\right)}\right] .
$$

Substituting this into Eq. (39) for $\bar{Q}$, we find that the Lifshitz line coincides with the line $\alpha=0$. The magnetization $M$ varies continuously along this line.

The evolution of the order parameters $M$ and $Q$ in the vicinity of the first-order transition from the paramagnet into the spiral state are plotted in Fig. 5. We see that the jumps in $M$ and $Q$ get smaller as we approach the tricritical point.

Previous analyses ${ }^{21}$ determined the phase diagram of spiral and uniform ferromagnets (they did not consider a spin-nematic phase) by brute force numerical (Monte Carlo) evaluation of the fluctuation corrections to the free energy, Eq. (8), at a given $g$ and $T$, for different values of $M$ and $Q$. We, instead, evaluate the phase diagram within a Ginzburg-Landau expansion and obtain an analytical approximation at low temperatures. The agreement between the two methods is good in the vicinity of the tricritical point where the expansion is controlled. In addition, we were able to determine the location of the boundary between the uniform and the spiral ferromagnets as well as the nature of this transition. 


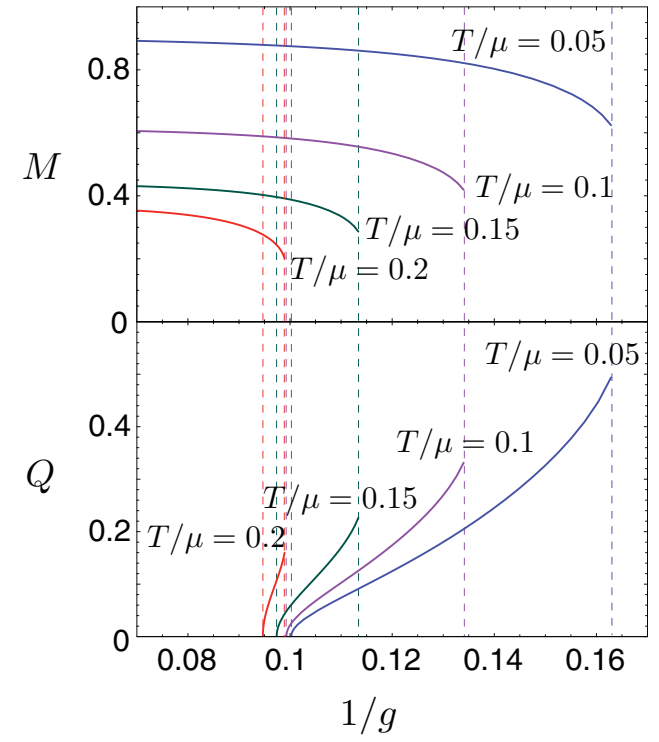

FIG. 5. (Color online) The evolution of the order parameters $M$ and $Q$ in the spiral phase. As we approach the tricritical point, the jumps in $M$ and $Q$ along the line of first-order transitions become smaller. At the tricritical point, $M=Q=0$. At the Lifshitz transition between the uniform ferromagnet and the spiral ferromagnet, $Q$ goes continuously to zero, while $M$ remains finite and behaves smoothly.

\section{Fluctuation-driven spin nematic}

Finally, we determine the region of the phase diagram where the $d$-wave spin-nematic phase has the lowest free energy. By analyzing the free energy, Eq. (35), we find that, for temperatures below $T=0.02 \mu$, the transition from the paramagnet into the spiral state is preempted by a transition into a spin-nematic state. The line of first-order transitions between the paramagnet and the spin nematic is given by the equation,

$$
\beta^{2}=4 \frac{\left\langle d_{\mathbf{k}}^{2}\right\rangle\left\langle d_{\mathbf{k}}^{6}\right\rangle}{\left\langle d_{\mathbf{k}}^{4}\right\rangle^{2}}(g-\alpha) \gamma
$$

From the evaluation of this equation for spin-nematic states with $d$ - and $p$-wave symmetries, we find that the instability for the formation of the $d$-wave spin nematic occurs at slightly higher temperatures and is, therefore, favored. This, however, might change, with dimensionality, the form of the electron dispersion or the range of the interactions.

Comparing the free energies of the spiral and spin-nematic phases, we find that the spin-nematic state penetrates into the region where the spiral state was previously favored. The details of the transition between the spiral and the spin-nematic phases are potentially very interesting but are hard to analyze. Introducing phase slips into the spiral restores translational symmetry and renders the phase nematic. Whether this is indeed the spin-nematic phase identified here or something more exotic ${ }^{34}$ is not clear.

The phase diagram, including the spin-nematic state is shown in Fig. 6. Note that this phase diagram is plotted on a logarithmic scale since the spin-nematic state onsets at a temperature, which is an order of magnitude lower than the temperature of the tricritical point where the spiral forms.

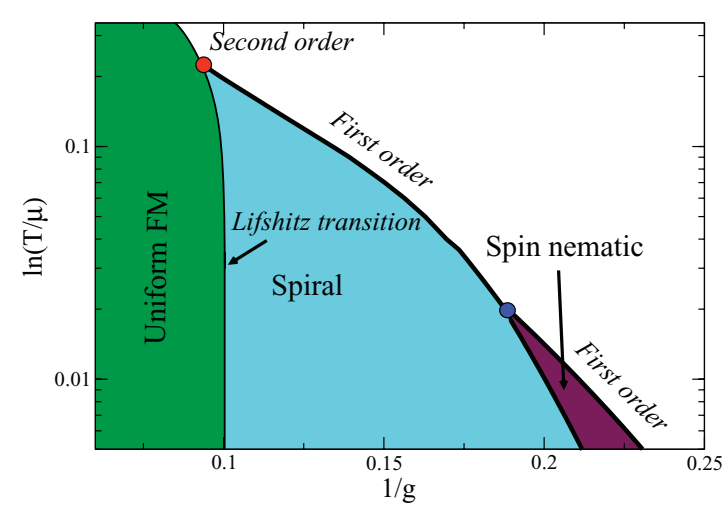

FIG. 6. (Color online) Phase diagram of the itinerant ferromagnet, allowing for the possibility for the formation of spiral and spinnematic phases. At temperatures, which are about an order of magnitude smaller than the temperature of the tricritical point, a $d$-wave spin nematic forms between the spiral ferromagnet and the paramagnet.

To summarize, quantum fluctuations have generated a coupling in the spin-nematic channel and have stabilized a spin-nematic phase. This is similar to the mechanism by which a superconducting state is stabilized in spin-fluctuation theory, ${ }^{27,28}$ and we anticipate that our approach can be applied to study superconductivity as well. We emphasize that the quantum order-by-disorder approach incorporates charge fluctuations on the same footing as spin fluctuations (see Sec. II B). As pointed out by Chubukov and Maslov, ${ }^{24}$ charge fluctuations are essential to mediate the formation of a spin-nematic state.

\section{Phase diagram in the presence of an anisotropic dispersion}

Changing from a free-electron dispersion to a band dispersion can have a profound effect upon the magnetic phase diagram. In the extreme, it can lead to nesting and the formation of modulated (antiferromagnetic) phases even in mean-field theory. Here, we consider the effect of a weakly anisotropic dispersion $\epsilon_{\mathbf{k}}=\frac{k^{2}}{2}+\delta \epsilon_{\mathbf{k}}$, with $\delta \epsilon_{\mathbf{k}}=\delta \cos (4 \phi) \sin \theta$. This deformation makes the dispersion more tight bindinglike. By changing the subtle balance between kinetic energy and fluctuation corrections, the regions occupied by the different phases and the nature (e.g., first or second order) of the transitions are altered.

\section{Uniform ferromagnet}

First, we investigate the changes to the phase diagram of the uniform ferromagnet. Summing Eqs. (15) and (37) evaluated at $Q=0$, we arrive at the following expression for the free energy:

$$
\begin{aligned}
& \tilde{\mathcal{F}}=\tilde{\alpha} M^{2}+\tilde{\beta} M^{4}+\tilde{\gamma} M^{6}, \\
& \tilde{\alpha}=\alpha+\left(6 \beta_{\mathrm{MF}}+\frac{g^{2}}{2} \frac{\partial^{2} \alpha_{\mathrm{fl}}}{\partial^{2} \mu^{2}}\right) \frac{\left\langle\delta \epsilon_{\mathbf{k}}^{2}\right\rangle}{g^{2}}, \\
& \tilde{\beta}=\beta+15 \gamma \frac{\left\langle\delta \epsilon_{\mathbf{k}}^{2}\right\rangle}{g^{2}}, \quad \tilde{\gamma}=\gamma .
\end{aligned}
$$

As in the case of the isotropic $k^{2}$ dispersion, we find that the transition between the uniform ferromagnet and the 


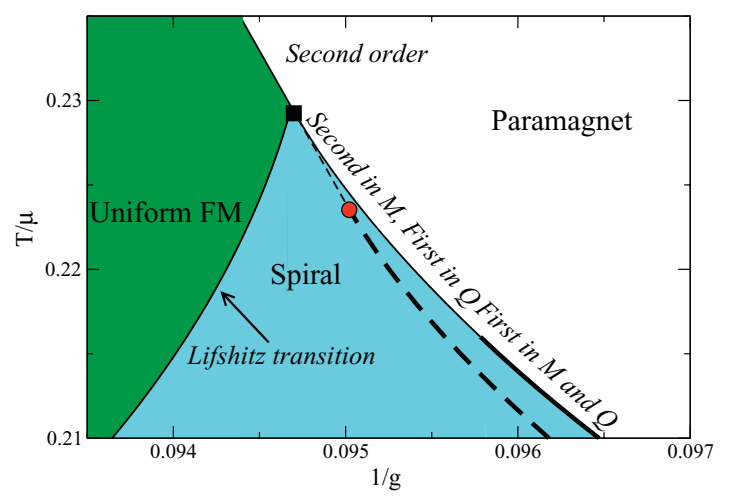

FIG. 7. (Color online) Modification to the phase diagram of the spiral state with a weakly anisotropic dispersion. The onset of the spiral no longer coincides with the putative tricritical point of the ferromagnet (circle). Instead, the spiral forms at a slightly higher temperature (square) and preempts a portion of the continuous transition between the uniform ferromagnet and the paramagnet (thin dashed line) as well as the first-order transition (thick dashed line). Note that, because of the anisotropy, the nature of the spiralto-paramagnet transition changes. At higher temperatures, $M$ now behaves continuously, while at low temperatures, the transition is first order in $M$ as in the case of an isotropic dispersion.

paramagnet is continuous at high temperatures and becomes first order at low temperatures due to a sign change in $\tilde{\beta}$. The line of second-order transitions between the uniform ferromagnet and the paramagnet is given by $\tilde{\alpha}=0$, while the line of first-order transitions is given by $\tilde{\beta}^{2}=4 \tilde{\alpha} \tilde{\gamma}$. The effect of the anisotropic correction to the dispersion is to slightly shift the locations of the phase boundaries, e.g., the temperature of the tricritical point is reduced to $T^{*}=0.225 \mu$ (see Fig. 7).

As we will see in the following, the effects of the anisotropy on the formation of the spiral and the nature of the transitions to the spiral ferromagnet are more interesting.

\section{Fluctuation-driven spiral}

Our analysis of the spiral phase follows the same steps as in the case of the isotropic free-electron dispersion in Sec. IV B 2. The resulting expressions are lengthy and are not particularly revealing in themselves. Therefore, we simply outline the main steps. The free energy of the spiral state is the sum of Eqs. (26) and (37) and is given by

$$
\begin{aligned}
\mathcal{F}[M, \mathbf{Q}]= & {\left[\tilde{\alpha}+\tilde{\beta}_{1}(\hat{\mathbf{Q}}) Q^{2}+\tilde{\gamma}_{1} Q^{4}\right] M^{2} } \\
& +\left(\tilde{\beta}+\tilde{\gamma}_{2} Q^{2}\right) M^{4}+\tilde{\gamma} M^{6},
\end{aligned}
$$

with $\tilde{\alpha}, \tilde{\beta}$, and $\tilde{\gamma}$ defined in Eq. (43), $\tilde{\gamma}_{1}=\gamma_{1}=\frac{3}{5} \gamma, \tilde{\gamma}_{2}=$ $\gamma_{2}=\gamma$, and

$$
\begin{aligned}
\tilde{\beta}_{1}(\hat{\mathbf{Q}})= & \frac{2}{3} \beta+2 \beta_{\mathrm{MF}}\left\langle\left(\frac{\mathbf{Q} \cdot \nabla \delta \epsilon_{\mathbf{k}}}{k_{F} Q}\right)^{2}\right\rangle \\
& +30 \frac{\gamma}{g^{2}}\left\langle\left(\frac{\mathbf{k} \cdot \mathbf{Q}}{k_{F} Q}\right)^{2} \delta \epsilon_{\mathbf{k}}^{2}\right\rangle .
\end{aligned}
$$

This free energy now depends upon the direction $\hat{\mathbf{Q}}=\mathbf{Q} / Q$ and is no longer invariant under rotations of the spiral. This is the consequence of the anisotropic dispersion which breaks the continuous rotation symmetry. It turns out that, for the particular anisotropy $\delta \epsilon_{\mathbf{k}}=\delta \cos (4 \phi) \sin \theta$, the free energy is minimized for spirals with $\hat{\mathbf{Q}}$ along the $z$ axis.

Notice that the proportionality between coefficients found in the case of the free-electron dispersion is broken by the anisotropic dispersion. For example, the coefficient of the term $Q^{2} M^{2}$ is no longer proportional to that of the $M^{4}$ term. This broken proportionality changes the nature of the transition between the spiral ferromagnet and the paramagnet (see Fig. 7) as well as the relative size of the regions occupied by different phases.

a. Optimum wave vector. The optimum wave vector is obtained by minimizing the free energy, Eq. (44), with respect to $Q$ for a given magnetization $M$. In this way, we obtain $\bar{Q} \equiv \bar{Q}[M]$. Substituting this value of $Q$ back into Eq. (44), we obtain the free energy at the optimum wave vector as a function of $M$,

$$
\tilde{\mathcal{F}}_{\bar{Q}}[M]=\tilde{\alpha}_{\bar{Q}} M^{2}+\tilde{\beta}_{\bar{Q}} M^{4}+\tilde{\gamma}_{\bar{Q}} M^{6},
$$

where $\tilde{\alpha}_{\bar{Q}}, \tilde{\beta}_{\bar{Q}}$, and $\tilde{\gamma}_{\bar{Q}}$ are the appropriate generalizations of the free-electron forms given in Eq. (40).

b. Paramagnet-to-spiral transition. As discussed in the case of the isotropic free-electron dispersion, in principle, there are two ways in which the system can make a transition from the paramagnet into the spiral state: (i) a second-order transition in $M$ (which occurs along the line $\tilde{\alpha}_{\bar{Q}}=0$ for $\tilde{\beta}_{\bar{Q}}>0$ ) and (ii) a first-order transition in $M$ (which occurs along the line $\tilde{\beta}_{\bar{Q}}^{2}=4 \tilde{\alpha}_{\bar{Q}} \tilde{\gamma}_{\bar{Q}}$ for $\tilde{\beta}_{\bar{Q}}<0$ and $\tilde{\alpha}_{\bar{Q}}>0$ ). For the free-electron dispersion, only the latter possibility occurred. Now that we have broken the proportionality between Ginzburg-Landau coefficients by allowing for an anisotropic dispersion, both possibilities exist.

(i) The transition begins as second order in $M$ (and first order in $Q$ ) (thin solid line in Fig. 7, extending below the square symbol). This line was not present in the phase diagram with free-electron dispersion because the quartic coefficient $\beta$ was negative in the region where the spiral existed. This line preempts a portion of the line of second-order phase transitions between the paramagnet and the uniform ferromagnet (thin dashed line) and the line of first-order transitions from the paramagnetic to the uniform ferromagnetic state (thick dashed line). The formation of the spiral phase is, therefore, no longer coincident with the putative tricritical point of the uniform ferromagnet (shown as a circle in Fig. 7) but sets in at higher temperatures as indicated by a square symbol in Fig. 7 .

(ii) The second-order transition between the paramagnet and the spiral gives way to a first-order transition at lower temperatures - shown as a thick solid line in Fig. 7.

c. Uniform ferromagnet-to-spiral transition. The boundary between the spiral and the uniform ferromagnetic phases remains a Lifshitz transition where the optimal wave vector falls continuously to zero and $M$ is continuous. In the case of the free-electron dispersion, this has turned out to be coincident with the $\alpha=0$ line. The situation is not so simple when we allow for an anisotropic dispersion. While the magnetization remains continuous, within our numerical resolution, we cannot exclude that the derivative of $M$ becomes discontinuous. The anisotropic dispersion has had two key effects upon the phase diagram. First, the region occupied by the spiral phase has increased, and second, the onset of the 
spiral is no longer coincident with the putative tricritical point of the uniform ferromagnet.

\section{Fluctuation-driven spin nematic}

The free energy of the $d$-wave spin-nematic state in the presence of a distortion is given by the sum of Eqs. (35) and (38). We can rewrite this in the form

$$
\tilde{\mathcal{F}}[N]=\tilde{\alpha}_{N} N^{2}+\tilde{\beta}_{N} N^{4}+\tilde{\gamma}_{N} N^{6},
$$

where

$$
\begin{aligned}
& \tilde{\alpha}_{N}=-\left\langle d_{\mathbf{k}}^{2}\right\rangle(\alpha-g)-\left(6 \frac{\beta_{\mathrm{MF}}}{g^{2}}+\frac{1}{2} \frac{\partial^{2} \alpha_{\mathrm{fl}}}{\partial \mu^{2}}\right)\left\langle\delta \epsilon_{\mathbf{k}}^{2} d_{\mathbf{k}}^{2}\right\rangle, \\
& \tilde{\beta}_{N}=\left\langle d_{\mathbf{k}}^{4}\right\rangle \beta+15 \frac{\gamma}{g^{2}}\left\langle\delta \epsilon_{\mathbf{k}}^{2} d_{\mathbf{k}}^{4}\right\rangle, \quad \tilde{\gamma}_{N}=\left\langle d_{\mathbf{k}}^{6}\right\rangle \gamma .
\end{aligned}
$$

The transition from the paramagnet into the $d$-wave spin nematic is first order and occurs when $\tilde{\beta}_{N}^{2}=4 \tilde{\alpha}_{N} \tilde{\gamma}_{N}$. The boundary between the spiral and the spin nematic is obtained by comparison of their free energies - although, as we stated above, the details of how this transition occurs may be subtle. When the dispersion is anisotropic, the spin-nematic state occurs at higher temperatures than in the case of the isotropic free-electron dispersion. This is because it becomes easier to redistribute the kinetic-energy cost of forming a spin nematic when the dispersion is anisotropic.

To summarize, we have used the Ginzburg-Landau expansion of the free energy, including quantum fluctuations, to determine the phase diagram of an itinerant ferromagnet in the vicinity of a quantum critical point. First, we investigated the effect of quantum fluctuations upon the phase diagram of the uniform ferromagnet. Below a certain temperature (the tricritical temperature), the paramagnet-to-ferromagnet transition becomes first order. Next, we allowed for the possibility of a spatially modulated ferromagnetic phase. For temperatures lower than the tricritical temperature $T^{*}$, it becomes energetically favorable to form a spiral state in between the paramagnetic and the uniform ferromagnetic states. The putative first-order transition between the paramagnet and the uniform ferromagnet is preempted by a transition into this spatially modulated state. Furthermore, we included the possibility of forming a $d$-wave spin-nematic state. This state is stabilized for temperatures below $T \approx 0.1 T^{*} \approx 0.02 \mu$ in between the paramagnetic and the spiral phases. Finally, we have shown that, in the presence of an anisotropic dispersion, both the spiral and the spin-nematic phases occupy a larger region of the phase diagram. Moreover, the onset of spiral order no longer coincides with the putative tricritical point of the uniform ferromagnet.

\section{CONCLUSIONS AND OUTLOOK}

We have shown how quantum fluctuations can lead to the formation of new phases in the vicinity of itinerant ferromagnetic quantum critical points. Quantum order by disorder not only provides an intuitive physical picture for this process, but also identifies a general principle ${ }^{7}$ behind the phase reconstruction near quantum critical points.

Quantum order by disorder is familiar in condensedmatter ${ }^{16-19}$ as well as in high-energy physics where it is referred to as the Coleman-Weinberg mechanism. ${ }^{35}$ In these familiar realizations, new ground states are stabilized by quantum fluctuations of a bosonic-order parameter. What makes our approach different is the immediate connection between Fermi-surface deformations (associated with the onset of competing order) and the enhancement of phase space available for low-energy quantum fluctuations.

Recently, it has been argued ${ }^{36}$ that the avoidance of naked quantum critical points due to the quantum order-bydisorder mechanism can be understood within the anti-de Sitter (AdS)/conformal-field theory correspondence ${ }^{37-39}$ between conformal-field theories, describing critical condensed-matter systems, and gravity in anti-de Sitter space. In the gravity context, the quantum critical state at finite temperatures corresponds with a Reissner-Nordstrom black hole in AdS space. It has been realized that such a black hole can become unstable at low temperatures and tends to collapse to a state with lower entropy. ${ }^{40}$ This entropic motive underlies the quantum order-by-disorder mechanism. Experimentally, the measurement of entropic landscapes has proven to be a revealing probe of the phase reconstruction near quantum critical points. $^{3,4}$

The fermionic quantum order-by-disorder approach presented in this paper not only establishes the connection to deformations of the Fermi surface, which are accessible by various experimental probes, but also leads to relatively simple analytical calculations, based on self-consistent second-order perturbation theory. As such, it is more accessible than technically involved diagrammatic techniques. ${ }^{10,12-15}$ The two approaches are formally equivalent; expanding self-consistently about a saddle point with the already established order resums a selected series of diagrams that give rise to nonanalytic corrections to the free energy.

We have used this approach to investigate fluctuation-driven phase reconstruction in the vicinity of an itinerant ferromagnetic quantum critical point in three spatial dimensions. This quantum critical point is unstable toward the formation of spiral and spin-nematic states. Quantum fluctuations would render the transition between the uniform ferromagnet and the paramagnet first order. However, this first-order transition is preempted by a modulated spiral ferromagnetic phase. At even lower temperatures, a $d$-wave spin-nematic state forms, which is slightly favored over a spin nematic with $p$-wave symmetry. It is located between the paramagnetic and the spiral phases. In order to describe more generic experimental systems, we determined the phase diagram in the presence of an anisotropic electron dispersion. The regions of phase space occupied by both the spiral and the spin-nematic phases are enlarged. Moreover, the onset of spiral order is no longer coincident with the putative tricritical point of the uniform ferromagnet, and the order of transitions is modified.

Similar spin-nematic instabilities near an itinerant ferromagnetic quantum critical point have been studied recently by Chubukov and Maslov ${ }^{24}$ within an extension to Hertz-Millis theory in a spin-fermion model. The authors point out that the inclusion of the effects of charge fluctuations (through Aslamov-Larkin corrections) in addition to spin fluctuations is crucial to mediate the formation of a spin nematic. Our quantum order-by-disorder approach incorporates charge fluctuations on the same footing as spin fluctuations. The results of Ref. 24 are very much in accord with those that 
we present here; a spin-nematic instability occurring out of the paramagnetic state is found to preempt the spiral phase. We note that the contact interaction used here, as opposed to the finite-range interaction in Ref. 24, lowers the free energy of the spiral relative to the spin nematic.

The quantum order-by-disorder approach can be applied to a variety of systems and phases. Adding a small spin-orbit coupling to the Stoner model of magnetism had previously enabled us to explain the partially ordered phase of $\mathrm{MnSi}$ (Ref. 6) in terms of quantum order by disorder. ${ }^{20}$ Work on the superconducting instability is in progress. It appears that our approach recovers the results of spin-fluctuation theory, thus, revealing the link between the spin-fluctuation theory used to treat superconducting instabilities and the extensions of Hertz-Millis theory that explain the instability of quantum critical points for other types of order.

There are several natural directions for developing our approach. The inclusion of superconducting instabilities is a priority - the nature of the superconducting phase where it overlaps with the spatially modulated magnetic phases raises the intriguing possibility of spontaneous fluctuation-driven modulated superconductivity. The calculations described in this paper are in three dimensions. Extending them to two dimensions, where the nonanalytic effects of fluctuations are even stronger, is an important step. ${ }^{41}$ Finally, the role of fluctuations near antiferromagnetic quantum critical points may also be susceptible to analysis using our methods.

\section{ACKNOWLEDGMENTS}

The authors benefited from stimulating discussions with D. Belitz, A. V. Chubukov, G. J. Conduit, B. D. Simons, and J. Zaanen. This work was supported by EPSRC under Grant No. EP/I 004831/1.

\section{APPENDIX A: FREE ENERGY OF THE UNIFORM FERROMAGNET IN TERMS OF MODIFIED PARTICLE-HOLE DENSITIES OF STATES}

The fluctuation corrections to the free energy are given by a high-dimensional integral over momenta $\mathbf{k}_{1}, \ldots, \mathbf{k}_{4}$ and correspond to excitations of virtual pairs of particle-hole pairs of opposite spin and equal and opposite momenta. It is, therefore, possible to rewrite the regularized fluctuation corrections $\mathcal{F}_{\text {fl }}$, Eq. (8), as a lower-dimensional integral over modified particle-hole densities of states,

$$
\mathcal{F}_{\mathrm{fl}}=2 g^{2} \sum_{\sigma= \pm 1} \int_{\mathbf{q}, \epsilon_{1}, \epsilon_{2}} \frac{\Delta \rho^{\sigma}\left(\mathbf{q}, \epsilon_{1}\right) \rho^{-\sigma}\left(-\mathbf{q}, \epsilon_{2}\right)}{\epsilon_{1}+\epsilon_{2}},
$$

where we have defined $\int_{\mathbf{q}}:=\int \frac{d^{3} \mathbf{q}}{(2 \pi)^{3}}$ and $\int_{\epsilon}:=\int_{-\infty}^{\infty} d \epsilon$. The modified particle-hole densities of states as a function of momentum $\mathbf{q}$ and energy $\epsilon$ are given by

$$
\begin{aligned}
\rho^{\sigma}(q, \epsilon) & =\int_{\mathbf{k}} n\left(\epsilon_{\mathbf{k}-\mathbf{q} / 2}^{\sigma}\right) \delta\left(\epsilon-\epsilon_{\mathbf{k}+\mathbf{q} / 2}^{\sigma}+\epsilon_{\mathbf{k}-\mathbf{q} / 2}^{\sigma}\right), \\
\Delta \rho^{\sigma}(q, \epsilon) & =\int_{\mathbf{k}} n\left(\epsilon_{\mathbf{k}-\mathbf{q} / 2}^{\sigma}\right) n\left(\epsilon_{\mathbf{k}+\mathbf{q} / 2}^{\sigma}\right) \delta\left(\epsilon-\epsilon_{\mathbf{k}+\mathbf{q} / 2}^{\sigma}+\epsilon_{\mathbf{k}-\mathbf{q} / 2}^{\sigma}\right),
\end{aligned}
$$

and are related to the particle-hole density of states as $\rho_{\mathrm{ph}}^{\sigma}=$ $\Delta \rho^{\sigma}-\rho^{\sigma}$. This form of the fluctuation correction will prove useful in our subsequent evaluation of the Ginzburg-Landau expansion of the uniform ferromagnet since the modified particle-hole density of states of the uniform ferromagnet can be calculated analytically. This leads to a tremendous simplification of the fluctuation integral.

The modified particle-hole densities of states are functions of the magnetization $M$, which enters through the dispersion $\epsilon_{\mathbf{k}}^{\sigma}=\epsilon_{\mathbf{k}}-\sigma g M$ of the uniform ferromagnet. We want to Taylor expand Eq. (A1) with respect to $M$. In doing so, we will require the derivatives of $\rho^{\sigma}$ and $\Delta \rho^{\sigma}$ with respect to $M$. However, since, in $\rho^{\sigma}$ and $\Delta \rho^{\sigma}$, the dispersion only enters for either spin up or spin down (and not both), we can relate the derivatives with respect to $M$ to derivatives with respect to the chemical potential $\mu$,

$$
\begin{aligned}
\left.\partial_{M}^{i} \Delta \rho^{\sigma}(q, \epsilon)\right|_{M=0} & =\left.(\sigma g)^{i} \partial_{\mu}^{i} \Delta \rho^{\sigma}(q, \epsilon)\right|_{M=0} \\
& =(\sigma g)^{i} \partial_{\mu}^{i} \Delta \rho(q, \epsilon) .
\end{aligned}
$$

Now, let us derive explicit expressions for $\Delta \rho=\left.\Delta \rho^{\sigma}\right|_{M=0}$ and $\rho=\left.\rho^{\sigma}\right|_{M=0}$ and their derivatives.

\section{Evaluation of $\rho(q, \epsilon)$ and $\Delta \rho(q, \epsilon)$}

For an isotropic free-energy dispersion, $\epsilon_{\mathbf{k}}=\frac{1}{2} k^{2}$ the angular integrals in $\Delta \rho(q, \epsilon)$ and $\rho(q, \epsilon)$ are easy to compute in three dimensions since the only angular dependencies enter through the volume element and the scalar product $\mathbf{k} \cdot \mathbf{q}=k q \cos \theta$. The remaining one-dimensional integrals over $k$ are elementary. The resulting modified particle-hole densities of states are given by

$$
\begin{aligned}
\rho(q, \epsilon)= & \frac{1}{(2 \pi)^{2}} \frac{T}{q} \ln \left(1+e^{-1 / T\left[\phi^{+}(\epsilon, q)-\mu\right]}\right), \\
\Delta \rho(q, \epsilon)= & \frac{1}{(2 \pi)^{2}} \frac{T}{q}\left[\frac{1}{1-e^{\epsilon / T}} \ln \left(1+e^{-1 / T\left[\phi^{-}(\epsilon, q)-\mu\right]}\right)\right. \\
& \left.+\frac{1}{1-e^{-\epsilon / T}} \ln \left(1+e^{-1 / T\left[\phi^{+}(\epsilon, q)-\mu\right]}\right)\right], \quad
\end{aligned}
$$

where

$$
\phi^{ \pm}(\epsilon, q)=\frac{1}{2}\left(\frac{\epsilon}{q} \pm \frac{q}{2}\right)^{2}
$$

For the derivatives of the modified densities of states with respect to the chemical potential, we obtain

$$
\begin{aligned}
\partial_{\mu}^{i} \rho(q, \epsilon) & =\frac{1}{(2 \pi)^{2}} \frac{1}{q} \partial_{\mu}^{(i-1)} n\left[\phi^{+}(\epsilon, q)\right], \\
\partial_{\mu}^{i} \Delta \rho(q, \epsilon) & =\frac{1}{(2 \pi)^{2}} \frac{1}{q} \partial_{\mu}^{(i-1)}\left\{n\left[\phi^{-}(\epsilon, q)\right] n\left[\phi^{+}(\epsilon, q)\right]\right\},
\end{aligned}
$$

where $n(x)=1 /\left[e^{(x-\mu) / T}+1\right]$ denotes the Fermi function.

\section{APPENDIX B: FLUCTUATION CONTRIBUTIONS TO $\alpha$ AND $\beta$}

Here, we outline the evaluation of the fluctuation contributions to the Ginzburg-Landau coefficients $\alpha$ and $\beta$ of the uniform ferromagnet,

$$
\alpha_{\mathrm{fl}}=\left.\frac{1}{2} \frac{\partial^{2} \mathcal{F}_{\mathrm{fl}}}{\partial M^{2}}\right|_{M=0} \quad \text { and } \quad \beta_{\mathrm{fl}}=\left.\frac{1}{4 !} \frac{\partial^{4} \mathcal{F}_{\mathrm{fl}}}{\partial M^{4}}\right|_{M=0} .
$$


The modified particle-hole densities of states and their derivatives with respect to the chemical potential were calculated in Appendix A. For brevity, we define the integrals,

$$
J_{i, j}=\int_{\mathbf{q}, \epsilon_{1}, \epsilon_{2}} \frac{\partial_{\mu}^{i} \Delta \rho\left(q, \epsilon_{1}\right) \partial_{\mu}^{j} \rho\left(q, \epsilon_{2}\right)}{\epsilon_{1}+\epsilon_{2}} .
$$

The fluctuation contributions to the free energy at zero magnetization $\mathcal{F}_{\mathrm{fl}}^{(0)}=\left.\mathcal{F}_{\mathrm{fl}}\right|_{M=0}$ are given by

$$
\mathcal{F}_{\mathrm{fl}}^{(0)}=4 g^{2} J_{0,0} \text {. }
$$

We evaluated the integral $J_{0,0}$ numerically for finite temperatures and analytically at $T=0$. The analytical calculation at $T=0$ correctly reproduces the result of Abrikosov and Khalatnikov. ${ }^{42}$ By Taylor expanding the fluctuation corrections to the free energy, we obtain the fluctuation contributions to the Ginzburg-Landau coefficients,

$$
\begin{aligned}
& \alpha_{\mathrm{fl}}=2 g^{4} \sum_{i=0}^{2}(-1)^{i}\left(\begin{array}{l}
2 \\
i
\end{array}\right) J_{i, 2-i}, \\
& \beta_{\mathrm{fl}}=\frac{g^{6}}{6} \sum_{i=0}^{4}(-1)^{i}\left(\begin{array}{l}
4 \\
i
\end{array}\right) J_{i, 4-i} .
\end{aligned}
$$

Some of the integrals $J_{i, j}$ are difficult to evaluate numerically, since at very low temperatures, the higher derivatives of the Fermi functions (which enter through the derivatives of modified particle-hole densities of states) are strongly peaked around the Fermi energy and rapidly change sign.

\section{Evaluation of $\alpha$}

From Eq. (B4), we see that we need to calculate three terms $J_{0,2}, J_{2,0}$, and $J_{1,1}$. In principle, $J_{0,2}, J_{2,0}$ are more difficult to calculate numerically (but possible) since they contain first derivatives of Fermi functions, which are strongly peaked. However, we can reduce the number of terms that we need to calculate by collecting some together to reexpress them as derivatives of $\mathcal{F}_{\mathrm{fl}}^{(0)}$ with respect to $\mu$. For example,

$$
\partial_{\mu}^{2} \mathcal{F}_{\mathrm{fl}}^{(0)}=4 g^{2}\left(J_{0,2}+2 J_{1,1}+J_{2,0}\right) .
$$

Using these relations, we can rewrite the fluctuation contribution to $\alpha$ as

$$
\begin{aligned}
\alpha_{\mathrm{fl}} & =2 g^{4}\left(J_{0,2}-2 J_{1,1}+J_{2,0}\right) \\
& =\frac{g^{2}}{2 !} \partial_{\mu}^{2} \mathcal{F}_{\mathrm{fl}}^{(0)}-8 g^{4} J_{1,1} .
\end{aligned}
$$

The remaining integral $J_{1,1}$ is easy to evaluate numerically. The temperature dependence of $\alpha_{\mathrm{fl}}$ is shown in Fig. 8. At zero temperature, we obtain

$$
\alpha_{\mathrm{fl}} \simeq-\lambda(1+2 \ln 2) g^{4},
$$

where $\lambda=\frac{16 \sqrt{2}}{3(2 \pi)^{6}}$.

\section{Evaluation of $\beta$}

In order to evaluate $\beta_{\mathrm{fl}}$, we need to evaluate five terms in Eq. (B4). As they contain higher-order derivatives of Fermi functions, they are even more difficult to evaluate numerically.

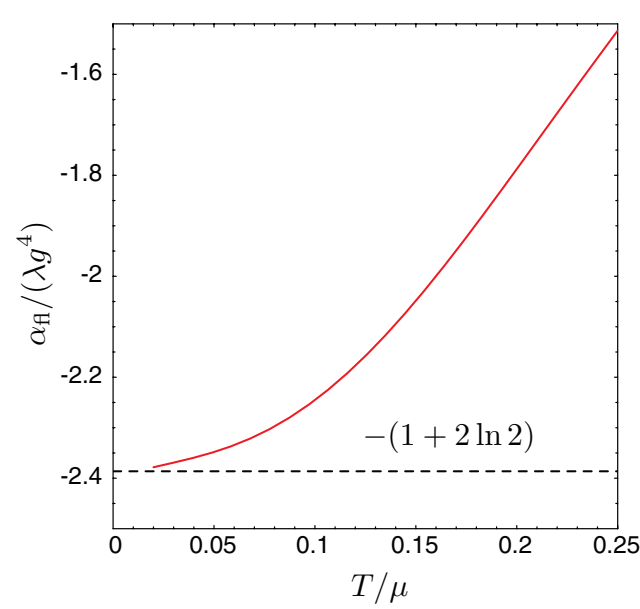

FIG. 8. (Color online) Fluctuation contributions to $\alpha$ : comparison of numerics (solid line) with leading low-temperature analytical dependence (dashed line).

We collect some of the terms together by noting that

$$
\partial_{\mu}^{4} \mathcal{F}_{\mathrm{fl}}^{(0)}=4 g^{2} \sum_{i=0}^{4}\left(\begin{array}{l}
4 \\
i
\end{array}\right) J_{i, 4-i},
$$

to get

$$
\beta_{\mathrm{fl}}=\frac{g^{4}}{4 !} \partial_{\mu}^{4} \mathcal{F}_{\mathrm{fl}}^{(0)}-\frac{4 g^{6}}{3}\left(J_{1,3}+J_{3,1}\right) .
$$

In this way, we avoid the explicit calculation of integrals $J_{0,4}$ and $J_{4,0}$, which contain third derivatives of Fermi functions. We can further simplify by noting that

$$
\partial_{\mu}^{2} J_{1,1}=J_{3,1}+2 J_{2,2}+J_{1,3} .
$$

If we reexpress $J_{3,1}+J_{1,3}$ in Eq. (B9) using Eq. (B10), we obtain

$$
\beta_{\mathrm{fl}}=\frac{g^{4}}{4 !} \partial_{\mu}^{4} \mathcal{F}_{\mathrm{fl}}^{(0)}-\frac{4}{3} g^{6} \partial_{\mu}^{2} J_{1,1}+\frac{8}{3} g^{6} J_{2,2} .
$$

We have already calculated the functions $\mathcal{F}_{\mathrm{fl}}^{(0)}$ and $J_{1,1}$ when we evaluated $\alpha_{\mathrm{ff}}$. Both are smooth functions, and the numerical evaluation of the derivatives with respect to $\mu$ is trivial. The

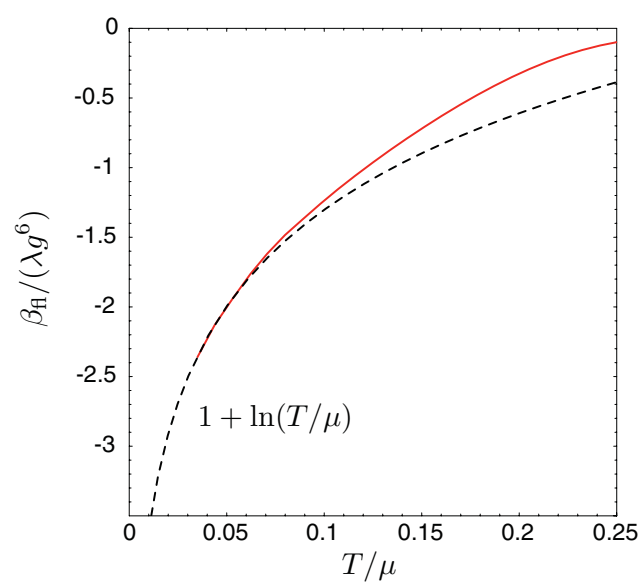

FIG. 9. (Color online) Fluctuation contributions to $\beta$ : comparison of numerics (solid line) with leading low-temperature analytical dependence (dashed line). 
leading temperature dependence of $\beta_{\mathrm{fl}}$ comes from the $J_{2,2}$ integral, which diverges as $T \rightarrow 0$. In this limit,

$$
\beta_{\mathrm{fl}} \simeq \lambda\left(1+\ln \frac{T}{\mu}\right) g^{6} .
$$

The logarithmic divergence with temperature arises from particle-hole pairs with momenta $q \approx 2 k_{F}$. The good agreement between our numerical and analytical results in the low-temperature regime is shown in Fig. 9.
${ }^{1}$ S. S. Saxena, P. Agarwal, K. Ahilan, F. M. Grosche, R. K. W. Haselwimmer, M. J. Steiner, E. Pugh, I. R. Walker, S. R. Julian, P. Monthoux et al., Nature (London) 406, 587 (2000).

${ }^{2}$ A. Huxley, I. Sheikin, E. Ressouche, N. Kernavanois, D. Braithwaite, R. Calemczuk, and J. Flouquet, Phys. Rev. B 63, 144519 (2001).

${ }^{3}$ R. A. Borzi, S. A. Grigera, J. Farrell, R. S. Perry, S. J. S. Lister, S. L. Lee, D. A. Tennant, Y. Maeno, and A. P. Mackenzie, Science 315, 214 (2007).

${ }^{4}$ A. W. Rost, R. S. Perry, J.-F. Mercure, A. P. Mackenzie, and S. A. Grigera, Science 325, 1360 (2009).

${ }^{5}$ M. Uhlarz, C. Pfleiderer, and S. M. Hayden, Phys. Rev. Lett. 93, 256404 (2004).

${ }^{6}$ C. Pfleiderer, D. Reznik, L. Pintschovius, H. v. Löhneysen, M. Garst, and A. Rosch, Nature (London) 414, 427 (2004).

${ }^{7}$ R. B. Laughlin, G. G. Lonzarich, P. Monthoux, and D. Pines, Adv. Phys. 50, 361 (2001).

${ }^{8}$ J. A. Hertz, Phys. Rev. B 14, 1165 (1976).

${ }^{9}$ A. J. Millis, Phys. Rev. B 48, 7183 (1993).

${ }^{10}$ D. Belitz, T. R. Kirkpatrick, and T. Vojta, Phys. Rev. B 55, 9452 (1997).

${ }^{11}$ T. Vojta, D. Belitz, T. R. Kirkpatrick, and R. Narayanan, Ann. Phys. 8, 593 (1999).

${ }^{12}$ J. Betouras, D. Efremov, and A. Chubukov, Phys. Rev. B 72, 115112 (2005).

${ }^{13}$ J. Rech, C. Pépin, and A. V. Chubukov, Phys. Rev. B 74, 195126 (2006).

${ }^{14}$ D. V. Efremov, J. J. Betouras, and A. Chubukov, Phys. Rev. B 77, 220401(R) (2008).

${ }^{15}$ D. L. Maslov and A. V. Chubukov, Phys. Rev. B 79, 075112 (2009).

${ }^{16}$ F. Mila, D. Poilblanc, and C. Bruder, Phys. Rev. B 43, 7891 (1991).

${ }^{17}$ J. Zaanen, Phys. Rev. Lett. 84, 753 (2000).

${ }^{18}$ J. Zaanen, O. Osman, H. Kruis, Z. Nussinov, and J. Tworzydlo, Philos. Mag. B 81, 1485 (2001).

${ }^{19}$ F. Krüger and S. Scheidl, Europhys. Lett. 74, 896 (2006).

${ }^{20}$ F. Krüger, U. Karahasanovic, and A. G. Green, Phys. Rev. Lett. 108, 067003 (2012).
${ }^{21}$ G. J. Conduit, A. G. Green, and B. D. Simons, Phys. Rev. Lett. 103, 207201 (2009).

${ }^{22}$ J. E. Hirsch, Phys. Rev. B 41, 6820 (1990).

${ }^{23}$ C. Wu, K. Sun, E. Fradkin, and S.-C. Zhang, Phys. Rev. B 75, 115103 (2007)

${ }^{24}$ A. V. Chubukov and D. L. Maslov, Phys. Rev. Lett. 103, 216401 (2009).

${ }^{25}$ R. K. Pathria, Statistical Mechanics (Butterworth-Heinemann, Oxford/Boston, 1996).

${ }^{26}$ G. J. Conduit and B. D. Simons, Phys. Rev. A 79, 053606 (2009).

${ }^{27}$ P. W. Anderson and W. F. Brinkman, Phys. Rev. Lett. 30, 1108 (1973).

${ }^{28}$ D. Fay and J. Appel, Phys. Rev. B 22, 3173 (1980).

${ }^{29}$ C. Pfleiderer, G. J. McMullan, S. R. Julian, and G. G. Lonzarich, Phys. Rev. B 55, 8330 (1997).

${ }^{30}$ C. Pfleiderer, S. R. Julian, and G. G. Lonzarich, Nature (London) 414, 427 (2001).

${ }^{31}$ A. Huxley, I. Sheikin, and D. Braithwaite, Physica B 284-288, 1277 (2000).

${ }^{32}$ W. Yu, F. Zamborszky, J. D. Thompson, J. L. Sarrao, M. E. Torelli, Z. Fisk, and S. E. Brown, Phys. Rev. Lett. 92, 086403 (2004).

${ }^{33}$ M. Otero-Leal, F. Rivadulla, S. S. Saxena, K. Ahilan, and J. Rivas, Phys. Rev. B 79, 060401(R) (2009).

${ }^{34}$ T. Senthil, S. Sachdev, and M. Vojta, Phys. Rev. Lett. 90, 216403 (2003).

${ }^{35}$ S. Coleman and E. Weinberg, Phys. Rev. D 7, 1888 (1973).

${ }^{36}$ J.-H. She, J. Zaanen, A. R. Bishop, and A. V. Balatsky, Phys. Rev. B 82, 165128 (2010).

${ }^{37}$ J. Maldacena, Adv. Theor. Math. Phys. 2, 231 (1998).

${ }^{38}$ E. Witten, Adv. Theor. Math. Phys. 2, 253 (1998).

${ }^{39}$ S. S. Gubser, I. R. Klebanov, and A. M. Polyakov, Phys. Lett. B 428, 105 (1998).

${ }^{40}$ S. A. Hartnoll, C. P. Herzog, and G. T. Horowitz, J. High Energy Phys. 12 (2008) 015.

${ }^{41}$ G. J. Conduit, Phys. Rev. A 82, 043604 (2010).

${ }^{42}$ A. A. Abrikosov and I. M. Khalatnikov, Sov. Phys. JETP 6, 888 (1958). 